\title{
Bounds on the Size of the Minimum Dominating Sets of Some Cylindrical Grid Graphs
}

\author{
Mrinal Nandi, ${ }^{1}$ Subrata Parui, ${ }^{2}$ and Avishek Adhikari ${ }^{3}$ \\ ${ }^{1}$ Department of Statistics, West Bengal State University, Barasat, North 24 Parganas 700126, India \\ ${ }^{2}$ IMBIC, AH 317, Salt Lake City, Calcutta 700091, India \\ ${ }^{3}$ Department of Pure Mathematics, University of Calcutta, 35 Ballygunge Circular Road, Calcutta 700019, India
}

Correspondence should be addressed to Avishek Adhikari; avishek.adh@gmail.com

Received 30 August 2013; Accepted 23 January 2014; Published 7 April 2014

Academic Editor: Liying Kang

Copyright (C) 2014 Mrinal Nandi et al. This is an open access article distributed under the Creative Commons Attribution License, which permits unrestricted use, distribution, and reproduction in any medium, provided the original work is properly cited.

Let $\gamma\left(P_{m} \square C_{n}\right)$ denote the domination number of the cylindrical grid graph formed by the Cartesian product of the graphs $P_{m}$, the path of length $m, m \geq 2$, and the graph $C_{n}$, the cycle of length $n, n \geq 3$. In this paper we propose methods to find the domination numbers of graphs of the form $P_{m} \square C_{n}$ with $n \geq 3$ and $m=5$ and propose tight bounds on domination numbers of the graphs $P_{6} \square C_{n}, n \geq 3$. Moreover, we provide rough bounds on domination numbers of the graphs $P_{m} \square C_{n}, n \geq 3$ and $m \geq 7$. We also point out how domination numbers and minimum dominating sets are useful for wireless sensor networks.

\section{Introduction}

The problem of domination is one of the most widely studied topics in graph theory: the 1998 book by Haynes et al. [1] contains a bibliography with over 1200 papers on the subject. The domination problem was studied from the $1950 \mathrm{~s}$ onwards, but the rate of research on domination significantly increased in the mid-1970s.

The signed domination number of a graph $G$ was defined in [2] and has been studied by several authors including $[2,3]$. Independent dominating sets were introduced into the theory of games by Morgenstern in [4]. For an extensive survey of domination problems and comprehensive bibliography the readers are referred to [5]. The study of domination numbers of products of graphs was initiated by Vizing [6]. He conjectured that the domination number of the Cartesian product of two graphs is always greater than or equal to the product of the domination numbers of the two factors, a conjecture which is still unproven. In [7], a link is shown between the existence of tilings in Manhattan metric with $\{1\}$, bowls and minimum total dominating sets of Cartesian products of paths and cycles. Domination numbers of Cartesian products were intensively investigated in [7-9].

The graphs considered here are finite, nonempty, connected, undirected without loops and without multiple edges. Besides these, any undefined terms in this paper may be found in Harary [10].

Let $G$ be a simple graph whose vertex set and edge set are $V(G)$ and $E(G)$, respectively. The set $D \subseteq V(G)$ of a simple graph $G$ is called a dominating set if every vertex $v \in V(G) \backslash D$ is adjacent to some vertex $u \in D$. The domination number of the graph $G$ is the cardinality of a smallest dominating set of the graph $G$; it is usually denoted by $\gamma(G)$ and dominating set with smallest cardinality is called a minimum dominating set of the graph $G$.

For any two graphs $G$ and $H$, the Cartesian product $G \square H$ is the graph with vertex set $V(G) \times V(H)$ and with edge set $E(G \times H)$ such that $\left(u_{1}, v_{1}\right)\left(u_{2}, v_{2}\right) \in E(G \times H)$, whenever $v_{1}=v_{2}$ and $u_{1} u_{2} \in E(G)$, or $u_{1}=u_{2}$ and $v_{1} v_{2} \in E(H)$ [11].

In this paper we follow the following notations and terminologies. The numbers $0,1,2, \ldots, n-1$ always denote the vertices of a path $P_{n}$ or a cycle $C_{n}$. Let $\gamma\left(P_{m} \square P_{n}\right)$ and $\gamma\left(P_{m} \square C_{n}\right)$ 
denote the domination numbers of Cartesian product graphs $P_{m} \square P_{n}$ and $P_{m} \square C_{n}$, respectively. Let $(G)_{v}=G \times\{v\}$, where $v \in V(H)$ and $(H)_{u}=\{u\} \times H$, where $u \in V(G) .(G)_{v}$ and $(H)_{u}$ are called the layers of $G$ and $H$, respectively. Moreover, layer of a dominating set means $D \cap\left(P_{m}\right)_{i}$ for $i \in V\left(C_{n}\right)$. Next we shall define the term modified concatenation of two dominating sets of $P_{m} \square C_{n_{1}}$ and $P_{m} \square C_{n_{2}}$. If $D_{1}$ and $D_{2}$ are two dominating sets of $P_{m} \square C_{n_{1}}$ and $P_{m} \square C_{n_{2}}$, respectively, then the modified concatenation of $D_{1}$ and $D_{2}$ is a subset $D$ of $P_{m} \square C_{n_{1}+n}$ such that $\mathrm{D} \cap\left(P_{m}\right)_{i}=D_{1} \cap\left(P_{m}\right)_{i}, i=0,1, \ldots, n_{1}-1$, and $D \cap\left(P_{m}\right)_{n_{1}+i}=D_{2} \cap\left(P_{m}\right)_{i}, i=0,1, \ldots, n_{2}-1$; that is, the $i$ th $\left(P_{m}\right)$-layer of $D$ is coming from the $i$ th $\left(P_{m}\right)$-layer of $D_{1}$ if $0 \leq i \leq n_{1}-1$ and from the $i$ th $\left(P_{m}\right)$-layer of $D_{2}$ if $n_{1} \leq i \leq n_{1}+n_{2}-1$. The illustration of modified concatenation is shown in Figure 1.

One of the most challenging problems concerning the domination number of Cartesian products of graphs is the proof of the Vizing Conjecture, namely, $\gamma(G \square H) \geq$ $\gamma(G) \gamma(H)$ [6]. Despite numerous results showing its validity in some special cases, till date the conjecture remains an open problem. Partial works have been made towards finding the domination numbers of some particular Cartesian products. This problem also seems to be difficult one and the authors of [12] proved that even for subgraphs of $P_{m} \square P_{n}$, this problem is NP-complete. In [13], Jacobson and Kinch established the following results:

For all $n \geq 1$,

(i) $\gamma\left(P_{2} \square P_{n}\right)=\lfloor(n+2) / 2\rfloor$.

(ii) $\gamma\left(P_{3} \square P_{n}\right)=\lfloor(3 n+4) / 4\rfloor$.

(iii)

$\gamma\left(P_{4} \square P_{n}\right)= \begin{cases}n+1, & \text { for } n=1,2,3,5,6,9 ; \\ n, & \text { otherwise. }\end{cases}$

In [14], Chang and Clark established the following results:

(i)

$$
\gamma\left(P_{5} \square P_{n}\right)= \begin{cases}\left\lfloor\frac{6 n+6}{5}\right\rfloor, & \text { for } n=2,3,7 ; \\ \left\lfloor\frac{6 n+8}{5}\right\rfloor, & \text { otherwise. }\end{cases}
$$

(ii)

$$
\begin{aligned}
& \gamma\left(P_{6} \square P_{n}\right) \\
& = \begin{cases}\left\lfloor\frac{10 n+10}{7}\right\rfloor, & \text { for } n \geq 6 \text { and } n \equiv 1(\bmod 7) ; \\
\left\lfloor\frac{10 n+12}{7}\right\rfloor, & \text { otherwise if } n \geq 4 .\end{cases}
\end{aligned}
$$

In [11], the authors established the following results regarding the Cartesian product of two cycles.

(i) For $n \geq 4, \gamma\left(C_{3} \square C_{n}\right)=n-\lfloor n / 4\rfloor$.

(ii) For $n \geq 4, \gamma\left(C_{4} \square C_{n}\right)=n$.

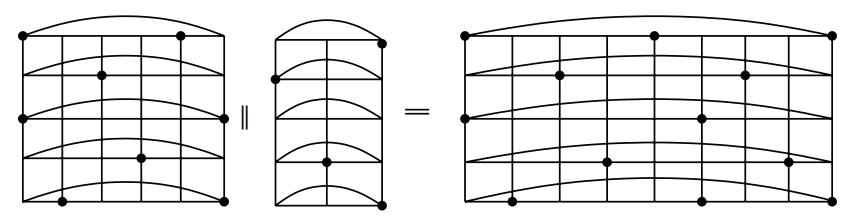

Figure 1: Modified concatenation of dominating sets of $P_{5} \square C_{6}$ and $P_{5} \square C_{3}$ to get dominating set for $P_{5} \square C_{9}$.

(iii) For $n \geq 5$,

$$
\gamma\left(C_{5} \square C_{n}\right)= \begin{cases}n, & n=5 k ; \\ n+1, & n \in\{5 k+1,5 k+2,5 k+4\} .\end{cases}
$$

Furthermore, $\gamma\left(C_{5} \square C_{5 k+3}\right) \leq 5(k+1)$.

More works may be found in $[8,9,15,16]$.

In the paper [17], Nandi et al. dealt with the domination number of some special types of graphs, known as cylindrical grid graphs $P_{m} \square C_{n}, m \geq 2, n \geq 3$ as shown in Figure 2 . An alternative way of looking at the same cylindrical grid graph is also shown in Figure 2, where the leftmost column in all figures denotes the layer $\left(P_{m}\right)_{0}$. In that paper the authors found the domination numbers as well as minimum dominating sets of the graphs $P_{m} \square C_{n}$, for $m=2,3,4$ and for all $n \geq 3$ and provided bounds on $\gamma\left(P_{m} \square C_{n}\right)$ for $m=5$ and for all $n \geq 3$. They pose an open problem for finding the domination numbers of the $P_{m} \square C_{n}$, for $m \geq 5$.

In the current paper, we deal with the above-mentioned open problem as posed in [17] and towards solving the problem, we get some partial results. We find the domination numbers as well as minimum dominating sets of the graphs $P_{m} \square C_{n}$, for $m=5$ and for all $n \geq 3$. We also give tight bounds on $\gamma\left(P_{m} \square C_{n}\right)$ for $m=6$ and for all $n \geq 3$. Moreover, we provide rough bounds on domination numbers of the graphs $P_{m} \square C_{n}, n \geq 3$ and $m \geq 7$. We also point out how domination numbers and minimum dominating sets are useful for wireless sensor networks. As a brief summary, we state the following results that we prove in the subsequent sections.

For all $n \geq 3$,

(i) $\gamma\left(P_{5} \square C_{3}\right)=4$ and for $n \geq 4, \gamma\left(P_{5} \square C_{n}\right)=n+$ $\lfloor n / 5\rfloor+p_{n}$, where

$$
p_{n}= \begin{cases}0, & \text { if } n \equiv 0(\bmod 10) \\ 2, & \text { if } n \equiv 3,9(\bmod 10) \\ 1, & \text { otherwise. }\end{cases}
$$

(ii) $\gamma\left(P_{6} \square C_{3}\right)=5, \gamma\left(P_{6} \square C_{4}\right)=6, \gamma\left(P_{6} \square C_{5}\right)=8$, $\gamma\left(P_{6} \square C_{6}\right)=9, \gamma\left(P_{6} \square C_{7}\right)=11$, and for $n>7$, 


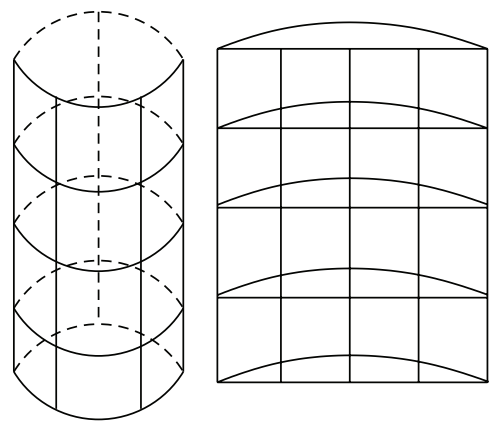

Figure 2: Two different looks of the cylindrical grid graph $P_{5} \square C_{5}$.

$$
\begin{aligned}
\frac{4 n}{3} & \leq \gamma\left(P_{6} \square C_{n}\right) \\
& \leq \begin{cases}\left\lceil\frac{10 n}{7}\right\rceil, & \text { for } n \equiv 0,4,5,8,10,11,12(\bmod 14) ; \\
\left\lceil\frac{10 n}{7}\right\rceil+1, & \text { otherwise. }\end{cases}
\end{aligned}
$$

(iii) If $m=5 p+k$ and $n=5 q+l$, where $p, q$ are natural numbers and $k, l=0,1,2,3,4$, then

$$
\begin{aligned}
\left\lceil\frac{m n}{5}\right\rceil & =5 p q+k q+p l+\left\lceil\frac{k l}{5}\right\rceil \leq \gamma\left(P_{m} \square C_{n}\right) \\
& \leq \begin{cases}5 p q+(k+2) q+(l+1) p, & \text { if } l \neq 0 \\
5 p q+(k+2) q, & \text { if } l=0 .\end{cases}
\end{aligned}
$$

\section{Finding Minimum Dominating Sets of $P_{5} \square C_{n}$, for All $n \geq 3$}

In this section we find the domination numbers as well as minimum dominating sets of particular cylindrical grid graphs of the form $P_{m} \square C_{n}$, for all $n \geq 3$ and for $m=5$. To prove the main results we state the following lemmas and theorems that are proved in [17]. Throughout the paper we use the arithmetic operations of the indices over modulo $n$.

Lemma 1 (see [17]). Let $m \geq 2$. Then there exists a minimum dominating set $D$ of $P_{m} \square C_{n}$ such that for every $i \in V\left(C_{n}\right)$, $\left|\left(P_{m}\right)_{i} \cap D\right| \leq m-1$.

Lemma 2 (see [17]). There cannot be two consecutive $P_{m^{-}}$ layers having empty intersection with a minimum dominating set of $P_{m} \square C_{n}$, for $m \geq 3$ and $n \geq 4$.

Lemma 3 (see [17]). For every dominating set $D$ of $P_{m} \square C_{n}$, the following $m$ inequalities hold:

$$
x_{i-1}+3 x_{i}+x_{i+1} \geq n, \quad \forall i=0, \ldots, m-1,
$$

where $x_{i}=\left|\left(C_{n}\right)_{i} \cap D\right|$ for $i=0,1, \ldots, m-1$ and $x_{-1}=x_{m}=0$. Moreover, if $x_{i-1}+3 x_{i}+x_{i+1}=n$, then there does not exist any pair of vertices from $\left(\left(C_{n}\right)_{i-1} \cup\left(C_{n}\right)_{i} \cup\left(C_{n}\right)_{i+1}\right) \cap D$ such that they dominate a common vertex of $\left(C_{n}\right)_{i}$. Finally, $\sum_{i=0}^{m-1} x_{i}=$ $|D| \geq \gamma\left(P_{m} \square C_{n}\right)$.
Remark 4. The similar result holds for $x_{i}=\left|\left(P_{m}\right)_{i} \cap D\right|$.

Lemma 5 (see [17]). For $n \geq 3$, there exists a minimum dominating set $D$ of $P_{5} \square C_{n}$ such that for every $i \in V\left(C_{n}\right)$, $\left|\left(P_{5}\right)_{i} \cap D\right| \leq 3$.

Lemma 6 (see [17]). For $n \geq 5$, there exists a minimum dominating set $D$ of $P_{5} \square C_{n}$ such that for every $i \in V\left(C_{n}\right)$, either (a) $\left|\left(P_{5}\right)_{i} \cap D\right| \leq 2$ or (b) $\left|\left(P_{5}\right)_{i} \cap D\right|=3$ with $\left(P_{5}\right)_{i-1} \cap D=\phi$ and $\left(P_{5}\right)_{i+1} \cap D=\phi$ for $i \in V^{\prime}$ and $\left|\left(P_{5}\right)_{i} \cap D\right| \leq 2$ for all $i \notin V^{\prime}$, for some $V^{\prime} \subseteq V\left(C_{n}\right)$.

Lemma 7 (see [17]). For $n \geq 5$, there cannot be a dominating set $D$ with five consecutive $P_{5}$-layers having exactly one vertex in common with $D$.

Lemma 8 (see [17]). Let $D$ be a minimum dominating set with the property as stated in Lemma 6. Again let $\left(P_{5}\right)_{i}$ and $\left(P_{5}\right)_{j}$ be two layers having two vertices in common with $D$ and $\left|\left(P_{5}\right)_{i+1} \cap D\right| \neq 2,\left|\left(P_{5}\right)_{i+2} \cap D\right| \neq 2, \ldots,\left|\left(P_{5}\right)_{j-1} \cap D\right| \neq 2$. Then either $(a)\left|\left(P_{5}\right)_{i+1} \cap D\right|=0,\left|\left(P_{5}\right)_{i+2} \cap D\right|=3,\left|\left(P_{5}\right)_{i+3} \cap D\right|=0$, $\left|\left(P_{5}\right)_{i+4} \cap D\right|=3, \ldots,\left|\left(P_{5}\right)_{j-1} \cap D\right|=0$, or $(b) j-i \leq 5$ and $\left|\left(P_{5}\right)_{l} \cap D\right|=1$ for all $l=(i+1),(i+2), \ldots,(j-1)$.

Theorem 9 (see [17]). For $n \geq 6, \gamma\left(P_{5} \square C_{n}\right) \geq\lceil 6 n / 5\rceil$.

Note 1. Let us call the collection of $P_{5}$-layers $\left\{\left(P_{5}\right)_{k}: k=i+\right.$ $1, i+2, \ldots, j-1\}$ as a block, where $i$ and $j$ are as in Lemma 8 .

Then $|D|=(6 n / 5)+\left(\left(x_{3}+2\left(x_{3}-x_{2}^{\prime \prime}\right)+\left(4 x_{2}^{\prime}-x_{1}\right)+\right.\right.$ $\left.4 x_{2}^{0}\right) / 5$ ), where $x_{1}, x_{3}$ denotes the number of $P_{5}$-layers having 1 and 3 vertices, respectively, in common with $D, x_{2}^{\prime}$ denotes the number of blocks in which every $P_{5}$-layer has exactly one vertex in common with $D, x_{2}^{\prime \prime}$ denotes the number of blocks in which every $P_{5}$-layer has either 0 or 3 vertices in common with $D$, and $x_{2}^{0}$ denotes the number of blocks, where $j=i+1$, that is, when the block contains no $P_{5}$-layer.

The above note will be useful to prove Theorem 13 .

Theorem 10 (see [17]). Consider the following: 7

(a) $\gamma\left(P_{5} \square C_{3}\right)=4,(b) \gamma\left(P_{5} \square C_{4}\right)=5$, and (c) $\gamma\left(P_{5} \square C_{5}\right)=$

Using the above-mentioned lemmas and theorems, we are going to prove the following main results.

Theorem 11. For $n \geq 6, \gamma\left(P_{5} \square C_{n}\right) \leq n+\lfloor n / 5\rfloor+p_{n}$, where

$$
p_{n}= \begin{cases}0, & \text { if } n \equiv 0(\bmod 10) ; \\ 2, & \text { if } n \equiv 3,9(\bmod 10) ; \\ 1, & \text { otherwise. }\end{cases}
$$

Proof. Consider the dominating sets of $P_{5} \square C_{4}$ and $P_{5} \square C_{5}$ as in Theorem 10 and the dominating sets of $P_{5} \square C_{n}$ for $n=$ $6,7, \ldots, 13$ as shown in Figure 3.

Now using modified concatenation of the dominating set for $n=10$ repeatedly with suitably choosing one of these ten dominating sets, we get dominating sets beyond $n=13$. For example, to find a dominating set for $C_{5} \square P_{17}$, we use modified concatenation of the dominating set $C_{5} \square P_{10}$ with 
$C_{5} \square P_{7}$. Again to find a dominating set for $C_{5} \square P_{27}$, we use modified concatenation of the dominating set $C_{5} \square P_{10}$ with $C_{5} \square P_{17}$.

Corollary 12. For $n \geq 4$,

$$
\begin{aligned}
& \gamma\left(P_{5} \square C_{n}\right) \\
& = \begin{cases}n+\left\lfloor\frac{n}{5}\right\rfloor, & \text { if } n \equiv 0(\bmod 10) ; \\
n+\left\lfloor\frac{n}{5}\right\rfloor+1, & \text { if } n \equiv 1,2,4,6,7,8(\bmod 10) .\end{cases}
\end{aligned}
$$

Proof. Using Theorems 9, 10, and 11 we get the desired result.

Theorem 13. For $n \geq 5$,
(1) $\gamma\left(P_{5} \square C_{n}\right)=n+\lfloor n / 5\rfloor+1$, if $n \equiv 5(\bmod 10)$,
(2) $\gamma\left(P_{5} \square C_{n}\right)=n+\lfloor n / 5\rfloor+2$, if $n \equiv 3(\bmod 10)$,
(3) $\gamma\left(P_{5} \square C_{n}\right)=n+\lfloor n / 5\rfloor+2$, if $n \equiv 9(\bmod 10)$.

Proof. To prove the theorem we first recall Note 1 . Here for any minimum dominating set $D$ with the property of $P_{5} \square C_{n}$, as stated in Lemma 8 , we get $|D|=(6 n / 5)+\left(\left(x_{3}+2\left(x_{3}-x_{2}^{\prime \prime}\right)+\right.\right.$ $\left.\left.\left(4 x_{2}^{\prime}-x_{1}\right)+4 x_{2}^{0}\right) / 5\right)$, where the symbols are as in Note 1 .

(a) Let $n \equiv 5(\bmod 10)$; that is, $n=5(2 p+1), p=$ $0,1,2, \ldots$

We claim that for any minimum dominating set $D$ of $P_{5} \square C_{n}$ with the property as stated in Lemma 8, $x_{3}+2\left(x_{3}-\right.$ $\left.x_{2}^{\prime \prime}\right)+\left(4 x_{2}^{\prime}-x_{1}\right)+4 x_{2}^{0}>0$. Otherwise, there will exist a minimum dominating set $D$ of $P_{5} \square C_{n}$ with the same property such that $x_{3}+2\left(x_{3}-x_{2}^{\prime \prime}\right)+\left(4 x_{2}^{\prime}-x_{1}\right)+4 x_{2}^{0}=0$. Then $x_{3}=$ $x_{2}^{\prime \prime}=x_{2}^{0}=0$ and $4 x_{2}^{\prime}=x_{1}$ (since $x_{3} \geq x_{2}^{\prime \prime}, 4 x_{2}^{\prime} \geq x_{1}, x_{2}^{0} \geq 0$ ).

Hence $D$ has the following properties:

(1) each $P_{5}$-layers has exactly 1 or 2 vertices in common with $D$,

(2) each block has exactly $4 P_{5}$-layers; that is, without loss of generality we can write

$$
\left|\left(P_{5}\right)_{i} \cap D\right|=\left\{\begin{array}{lc}
2, & \text { if } i \equiv 0(\bmod 5) \\
1, & \text { otherwise. }
\end{array}\right.
$$

Since $\left|\left(P_{5}\right)_{5 k} \cap D\right|=2$ and $\left|\left(P_{5}\right)_{5 k+1} \cap D\right|=\mid\left(P_{5}\right)_{5 k+2} \cap$ $D|=|\left(P_{5}\right)_{5 k+3} \cap D|=|\left(P_{5}\right)_{5 k+4} \cap D \mid=1$ and $\mid\left(P_{5}\right)_{5 k+5} \cap$ $D \mid=2$, the number of vertices dominated by each of the vertices of $\left(P_{5}\right)_{5 k+2} \cap D$ and $\left(P_{5}\right)_{5 k+3} \cap D$ will be 4 (excluding themselves) and no vertex will be dominated by both of them simultaneously. Therefore $\bigcup_{j=0}^{5}\left[\left(P_{5}\right)_{5 k+j} \cap D\right]=$ $\{(0,5 k),(0,5 k+4),(1,5 k+2),(2,5 k),(2,5 k+5),(3,5 k+$ $3),(4,5 k+1),(4,5 k+5)\}$ or $\{(0,5 k+1),(0,5 k+5),(1,5 k+$ $3),(2,5 k),(2,5 k+5),(3,5 k+2),(4,5 k),(4,5 k+4)\}$ are the only two possibilities (as shown in Figure 4 ) of $\bigcup_{j=0}^{5}\left[\left(P_{5}\right)_{5 k+j} \cap D\right]$.

Now, without loss of generality let $(0,0),(2,0) \in D$. Then $(0,10 p),(2,10 p) \in D$.
Therefore $(1,10 p+2),(3,10 p+3) \in D$ and $(0,10 p+2) \notin$ $D ;(l, 10 p+3) \notin D$ for all $l=0,1,2,4$. Consider $(0,10 p+4) \epsilon$ $D$ and $(l, 10 p+4) \notin D$ for all $l=1,2,3,4$.

Hence $(4,10 p+4)$ cannot be dominated by the vertices of $D$, contradicting that $D$ is a dominating set.

Therefore $x_{3}+2\left(x_{3}-x_{2}^{\prime \prime}\right)+\left(4 x_{2}^{\prime}-x_{1}\right)+4 x_{2}^{0}>0$.

Hence for any minimum dominating set $D,|D|>6 n / 5=$ $6(2 p+1)$; that is, $|D| \geq 6(2 p+1)+1$. Therefore $\gamma\left(P_{5} \square C_{n}\right) \geq$ $6(2 p+1)+1=n+\lfloor n / 5\rfloor+1$. The rest of the proof of $(\mathrm{a})$ follows from Theorem 11 .

We note the following observation.

Observation 1. If $\left|\left(P_{5}\right)_{j} \cap D\right|=\left|\left(P_{5}\right)_{j+5} \cap D\right|=2,\left|\left(P_{5}\right)_{j+1} \cap D\right|=$ $\left|\left(P_{5}\right)_{j+2} \cap D\right|=\left|\left(P_{5}\right)_{j+3} \cap D\right|=\left|\left(P_{5}\right)_{j+4} \cap D\right|=1$, then (i) $(0, j)$ and $(2, j) \in D$ imply $(2, j+5),(4, j+5) \in D$ and (ii) $(2, j)$ and $(4, j) \in D$ imply $(0, j+5),(2, j+5) \in D$.

(b) Let $n \equiv 3(\bmod 10)$; that is, $n=10 p+3$.

We claim that $x_{3}+2\left(x_{3}-x_{2}^{\prime \prime}\right)+\left(4 x_{2}^{\prime}-x_{1}\right)+4 x_{2}^{0}>2$ for any minimum dominating set $D$ of $P_{5} \square C_{n}$ with the property as stated in Lemma 8.

If possible let there exist a minimum dominating set $D$ with the property as stated in Lemma 8 and $x_{3}+2\left(x_{3}-x_{2}^{\prime \prime}\right)+$ $\left(4 x_{2}^{\prime}-x_{1}\right)+4 x_{2}^{0}=0$. Then $x_{2}^{\prime \prime}=x_{3}=0$ and $x_{1}=4 x_{2}^{\prime}, x_{2}^{0}=0$ which will be impossible since $n \not \equiv 0(\bmod 5)$.

If possible let there exist a minimum dominating set $D$ with the property as stated in Lemma 8 and $x_{3}+2\left(x_{3}-x_{2}^{\prime \prime}\right)+$ $\left(4 x_{2}^{\prime}-x_{1}\right)+4 x_{2}^{0}=1$. Then $x_{2}^{\prime \prime}=x_{3}$ and $x_{2}^{0}=0$. Therefore $x_{2}^{\prime \prime}=x_{3}=1, x_{1}=4 x_{2}^{\prime}$, and $x_{2}^{0}=0$ or $x_{2}^{\prime \prime}=x_{3}=x_{2}^{0}=0$ and $x_{1}=4 x_{2}^{\prime}-1$.

Now if $x_{2}^{\prime \prime}=x_{3}=1$ and $x_{1}=4 x_{2}^{\prime}$ then let $x_{0}$ denote the number of $P_{5}$-layers having 0 vertices in common with $D$ and $x_{0}=x_{2}^{\prime \prime}+x_{3}=2$.

By Lemma $8, x_{2}=x^{0}+x_{2}^{\prime}+x_{2}^{\prime \prime}$ is the number of $P_{5}$-layers having 2 vertices in common with $D$ and $n=x_{0}+x_{1}+x_{2}+x_{3}=$ $x_{0}+x_{1}+x_{2}^{0}+x_{2}^{\prime}+x_{2}^{\prime \prime}+x_{3}=2+4 x_{2}^{\prime}+x_{2}^{\prime}+1+1=5 x_{2}^{\prime}+4 \not \equiv$ $3(\bmod 5)$ which is a contradiction.

Again if $x_{2}^{\prime \prime}=x_{3}=0$ and $x_{1}=4 x_{2}^{\prime}-1$ then $n=x_{0}+x_{1}+$ $x_{2}^{0}+x_{2}^{\prime}+x_{2}^{\prime \prime}+x_{3}=4 x_{2}^{\prime}-1+x_{2}^{\prime} \not \equiv 3(\bmod 5)$, which is also a contradiction.

If possible let there exist a minimum dominating set $D$ with the property as stated in Lemma 8 and $x_{3}+2\left(x_{3}-x_{2}^{\prime \prime}\right)+$ $\left(4 x_{2}^{\prime}-x_{1}\right)+4 x_{2}^{0}=2 \ldots(A)$. Then $x_{2}^{\prime \prime}=x_{3}$ (otherwise, $x_{3} \neq x_{2}^{\prime \prime}$ implies $x_{3}-x_{2}^{\prime \prime} \geq 1$ and $x_{3} \geq 1$, contradicting $\left.(A)\right)$ and $x_{2}^{0}=0$.

Now there are the following three cases.

Case 1. Consider $x_{3}=x_{2}^{\prime \prime}=x_{2}^{0}=0$ and $x_{1}=4 x_{2}^{\prime}-2$.

Since $x_{3}=x_{2}^{\prime \prime}=x_{2}^{0}=0$ each block contains only those $P_{5}$-layers which have exactly one vertex in common with $D$.

Again since $x_{1}=4 x_{2}^{\prime}-2$, there are two sub cases.

Subcase 1.1. When only one block contains two $P_{5}$-layers and other block contains four $P_{5}$-layers then without loss of generality let $\left|\left(P_{5}\right)_{j} \cap D\right|=2$ for $j=0,5,10, \ldots, 10 p$ and $\left|\left(P_{5}\right)_{j} \cap D\right|=1$, otherwise. Now $(0,0),(2,0) \in D$ or $(2,0),(4,0) \in D$. If possible let $(0,0),(2,0) \in D$. Then $(0,10 p),(2,10 p) \in D$ and $(j, 10 p) \notin D$ for $j=1,3,4$. But 

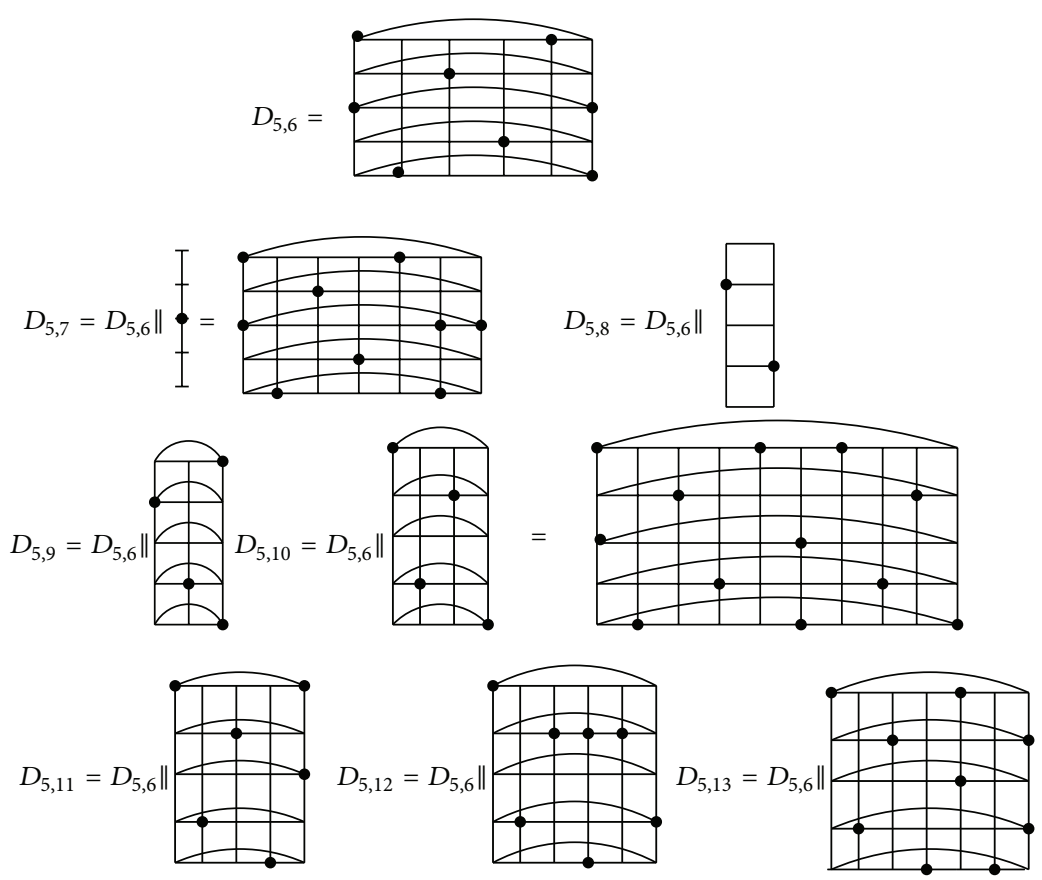

FIGURE 3: Figures showing a particular dominating set for $P_{5} \square C_{i}, i=6,7, \ldots, 13$, with $\left|D_{5,6}\right|=8,\left|D_{5,7}\right|=9,\left|D_{5,8}\right|=10,\left|D_{5,9}\right|=12$, $\left|D_{5,10}\right|=12,\left|D_{5,11}\right|=14,\left|D_{5,12}\right|=15$, and $\left|D_{5,13}\right|=17$.
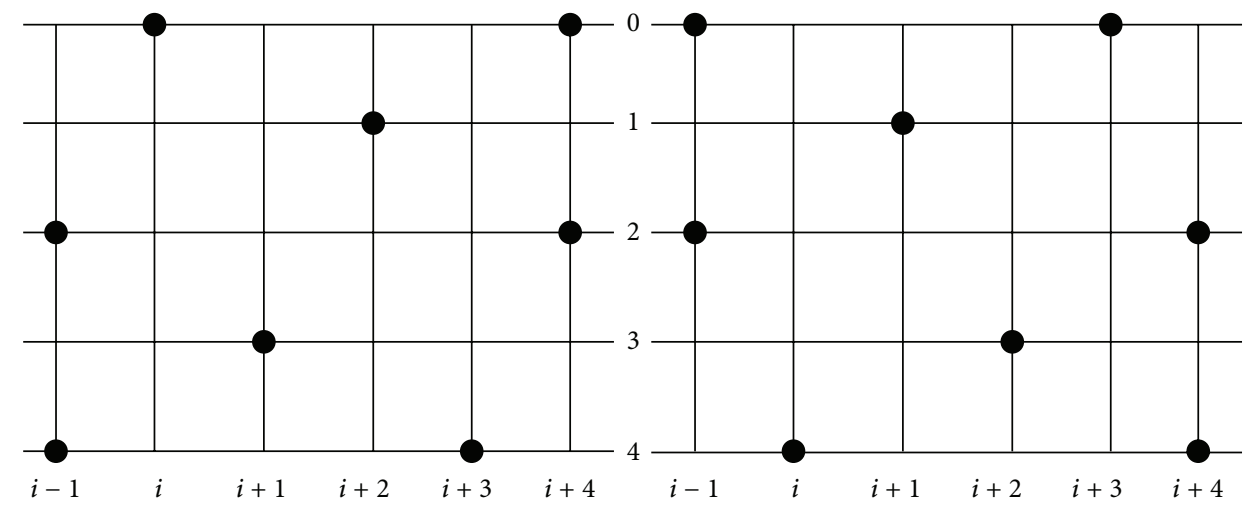

Figure 4: The only two possible configurations of the vertices of a minimum dominating set of the graph $P_{5} \square C_{n}$ in four consecutive layers $P_{i}, P_{i+1}, P_{i+2}$, and $P_{i+3}$.

this is not possible since $\left|\left(P_{5}\right)_{10 p+1} \cap D\right|=\left|\left(P_{5}\right)_{10 p+2} \cap D\right|=1$ and $(0,10 p+3),(2,10 p+3) \in D$ and $(j, 10 p+3) \notin D$ for $j=1,3,4$. Note that $\left(P_{5}\right)_{10 p+3}=\left(P_{5}\right)_{0}$.

Similar contradiction will be arrived for $(2,0),(4,0) \in D$.

Subcase 1.2. When only two blocks contain three $P_{5}$-layers and other blocks contain four $P_{5}$-layers then if

(i) the blocks containing three $P_{5}$-layers, each occurs consecutively then without loss of generality let $\left|\left(P_{5}\right)_{j} \cap D\right|=2$ for $j=0,5,10, \ldots, 10 p-5,10 p-1$ and $\left|\left(P_{5}\right)_{j} \cap D\right|=1$, otherwise. Now if $(0,0),(2,0) \in D$ then $(2,10 p-5),(4,10 p-5) \in D \Rightarrow(1,10 p+$ $1),(3,10 p-3) \in D \Rightarrow(0,10 p-1),(4,10 p-1) \in D$ which is a contradiction.Similar contradiction will be arrived for $(2,0),(4,0) \in D$,

(ii) the blocks containing three $P_{5}$-layers do not occur consecutively then among them one of the blocks consist of $j+1, j+2, j+3 P_{5}$-layer for some $j$ and either $(a)(0, j),(2, j) \in D$ and $(2, j+4),(4, j+4) \in D$ or $(b)(2, j),(4, j) \in D$ and $(0, j+4),(2, j+4) \in D$. In each case, we arrive at a contradiction. Hence we conclude that Case 1 cannot occur.

Case 2. Consider $x_{3}=x_{2}^{\prime \prime}=1, x_{2}^{0}=0$, and $x_{1}=4 x_{2}^{\prime}-1$.

In this case only two blocks contain three $P_{5}$-layers and other blocks contain four $P_{5}$-layers. Among these two blocks one contains only those $P_{5}$-layers which have exactly one 
vertex in common with $D$ and other block contains three layers whose number of vertices are $0,3,0$ consecutively.

Now we note the following observations.

Observation 2. If $\left|\left(P_{5}\right)_{j} \cap D\right|=\left|\left(P_{5}\right)_{j+4} \cap D\right|=2,\left|\left(P_{5}\right)_{j+2} \cap D\right|=$ 3 , and $\left|\left(P_{5}\right)_{j+1} \cap D\right|=\left|\left(P_{5}\right)_{j+3} \cap D\right|=0$ then $(0, j),(2, j) \in D$ implies $(0, j+4),(2, j+4) \in D$ and $(2, j),(4, j) \in D$ implies $(2, j+4),(4, j+4) \in D$.

Observation 3. If $\left|\left(P_{5}\right)_{j} \cap D\right|=\left|\left(P_{5}\right)_{j+4} \cap D\right|=2, \mid\left(P_{5}\right)_{j+1} \cap$ $D|=|\left(P_{5}\right)_{j+2} \cap D|=|\left(P_{5}\right)_{j+3} \cap D \mid=1$ then $(0, j),(2, j) \in D$ implies $(0, j+4),(2, j+4) \in D$ or $(0, j+4),(3, j+4) \in D$ and $(2, j),(4, j) \in D$ implies $(2, j+4),(4, j+4) \in D$ or $(1, j+4),(4, j+4) \in D$.

Without loss of generality we now assume that $\mid\left(P_{5}\right)_{0} \cap$ $D|=|\left(P_{5}\right)_{4} \cap D|=2,|\left(P_{5}\right)_{1} \cap D|=|\left(P_{5}\right)_{2} \cap D|=|\left(P_{5}\right)_{3} \cap D \mid=$ 1. Therefore by Observation 2 and Observation 1 in Case (a) we have either $(0,0),(2,0) \in D$ and $(2,4),(4,4) \in D$ or $(2,0),(4,0) \in D$ and $(0,4),(2,4) \in D$ which is again a contradiction by Observation 3 .

Case 3. Consider $x_{3}=x_{2}^{\prime \prime}=2, x_{2}^{0}=0$, and $x_{1}=4 x_{2}^{\prime}$.

In this case only two blocks contain three $P_{5}$-layers and other blocks contain four $P_{5}$-layers and these two blocks contain three layers whose number of vertices is $0,3,0$ consecutively. Therefore by the Observation 2, as in Case 2, we arrive at a contradiction.

Then $|D|=(6 n / 5)+\left(\left(x_{3}+2\left(x_{3}-x_{2}^{\prime \prime}\right)+\left(4 x_{2}^{\prime}-x_{1}\right)+4 x_{2}^{0}\right) / 5\right)>$ $((60 p+18) / 5)+(2 / 5)=12 p+4$.

Therefore $|D| \geq 12 p+5=10 p+3+2 p+2=n+\lfloor n / 5\rfloor+2$. This completes the proof of $(b)$.

(c) Let $n \equiv 9(\bmod 10)$; that is, $n=10 p+9$.

We claim that for any minimum dominating set $D$ of $P_{5} \square C_{n}$ with the property as stated in Lemma 8, $x_{3}+2\left(x_{3}-\right.$ $\left.x_{2}^{\prime \prime}\right)+\left(4 x_{2}^{\prime}-x_{1}\right)+4 x_{2}^{0}>1$.

If possible let $x_{3}+2\left(x_{3}-x_{2}^{\prime \prime}\right)+\left(4 x_{2}^{\prime}-x_{1}\right)+4 x_{2}^{0}=0$. Then as in (b), $n \neq \equiv 0(\bmod 5)$. As a result, we have the same contradiction.

If possible let $x_{3}+2\left(x_{3}-x_{2}^{\prime \prime}\right)+\left(4 x_{2}^{\prime}-x_{1}\right)+4 x_{2}^{0}=1$. Then $x_{3}=x_{2}^{\prime \prime}$ and therefore the following two cases arise.

Case 1. Consider $x_{3}=x_{2}^{\prime \prime}=x_{2}^{0}=0$ and $x_{1}=4 x_{2}^{\prime}-1$.

Case 2. Consider $x_{3}=x_{2}^{\prime \prime}=1, x_{1}=4 x_{2}^{\prime}$, and $x_{2}^{0}=0$.

In these two cases contradiction can be shown similarly as in $(\mathrm{b})$, where $n \equiv 3(\bmod 10)$.

Hence $|D|=(6 n / 5)+\left(\left(x_{3}+2\left(x_{3}-x_{2}^{\prime \prime}\right)+\left(4 x_{2}^{\prime}-x_{1}\right)+\right.\right.$ $\left.\left.4 x_{2}^{0}\right) / 5\right)>((60 p+54) / 5)+(1 / 5)=12 p+11$.

Therefore $|D| \geq 12 p+12=n+\lfloor n / 5\rfloor+2$. This completes the proof of (c).

Remark 14. For $n \geq 3$ and $n \neq 7$,

$$
\begin{aligned}
& \gamma\left(P_{5} \square C_{n}\right) \\
& \quad= \begin{cases}\gamma\left(P_{5} \square P_{n}\right)-1, & \text { when } n \equiv 0,2,4,7,8(\bmod 10) ; \\
\gamma\left(P_{5} \square P_{n}\right), & \text { otherwise, }\end{cases}
\end{aligned}
$$

and $\gamma\left(P_{5} \square C_{7}\right)=\gamma\left(P_{5} \square P_{7}\right)$. Moreover, for $n \geq 3, \gamma\left(P_{5} \square C_{n}\right)>$ $\gamma\left(P_{5}\right) \cdot \gamma\left(C_{n}\right)=2 \cdot\lceil n / 3\rceil$.

\section{Bounds on Domination Numbers of $P_{6} \square C_{n}$ for All $n \geq 3$}

In this section we give upper and lower bounds of the domination numbers of $P_{6} \square C_{n}$. Towards this direction, we first prove the following lemmas.

Lemma 15. Let $n \geq 4$. Then there exists a minimum dominating set $D$ of $P_{6} \square C_{n}$ with $\left|\left(P_{6}\right)_{i} \cap D\right| \leq 4 \forall i$.

Proof. Let $D$ be a minimum dominating set of $P_{6} \square C_{n}$ with the property $\left|\left(P_{6}\right)_{i} \cap D\right| \leq 5 \forall i$. Such a $D$ exists by Lemma 1 . Suppose further that $\left|\left(P_{6}\right)_{j} \cap D\right|=5$ for $k$ many $P_{6}$ layers.

Assume that $\left|\left(P_{6}\right)_{i} \cap D\right|=5$ for some $i$, then $\left|\left(P_{6}\right)_{i+1} \cap D\right| \leq$ 2; otherwise $D^{\prime}=D \backslash\left[\left(P_{6}\right)_{i} \cup\left(P_{6}\right)_{i+1}\right] \cup\{(0, i),(1, i+2),(2, i-$ $1),(3, i+1),(4, i-1),(5, i),(5, i+2)\}$ is a dominating set with $\left|D^{\prime}\right|<|D|$, which is a contradiction.

Similar contradiction shows that $\left|\left(P_{6}\right)_{i-1} \cap D\right| \leq 2$. Now construct $D^{\prime \prime}=D \backslash\left(P_{6}\right)_{i} \cup\{(0, i),(2, i-1),(2, i+1),(4, i),(5, i)\}$. Then $\left|D^{\prime \prime}\right| \leq|D|$ and hence $D^{\prime \prime}$ is a minimum dominating set with $k-1$ many $P_{6}$ layers having 5 vertices in common with $D^{\prime \prime}$. Repeating this construction we get the desired minimum dominating set and hence the lemma follows.

Lemma 16. For any minimum dominating set $D,\left|\left(P_{6}\right)_{i} \cap D\right|=$ $1=\left|\left(P_{6}\right)_{i+1} \cap D\right| \forall i$ implies $\left|\left(P_{6}\right)_{i+2} \cap D\right| \geq 2$ and $\left|\left(P_{6}\right)_{i-1} \cap D\right| \geq$ 2.

Proof. The proof of the lemma follows from Remark 4.

Lemma 17. For $n \geq 4$, there exists a minimum dominating set $D$ of $P_{6} \square C_{n}$ with the property (i) if $\left|\left(P_{6}\right)_{i} \cap D\right|=1,\left|\left(P_{6}\right)_{i+1} \cap D\right|=$ 1, $\left|\left(P_{6}\right)_{i+2} \cap D\right|=2$ for some $i$, then $\left|\left(P_{6}\right)_{i+3} \cap D\right| \geq 1$;

(ii) if $\left|\left(P_{6}\right)_{i} \cap D\right|=2,\left|\left(P_{6}\right)_{i+1} \cap D\right|=1,\left|\left(P_{6}\right)_{i+2} \cap D\right|=1$ for some $i$, then $\left|\left(P_{6}\right)_{i-1} \cap D\right| \geq 1$ and (iii) $\left|\left(P_{6}\right)_{i} \cap D\right| \leq 4 \forall i$.

Proof. Let $D$ be a minimum dominating set of $P_{6} \square C_{n}$ with the property $\left|\left(P_{m}\right)_{i} \cap D\right| \leq 4 \forall i$. Such a $D$ exists by Lemma 15 . Let $x_{i}=\left|\left(P_{6}\right)_{i} \cap D\right|, i=0,1, \ldots, n-1$.

When $n=4$, then $x_{i}=1, x_{i+1}=1$, and $x_{i+2}=2$ imply that $x_{i+3} \geq 1$ since by Remark $4, x_{i-1}+3 x_{i}+x_{i+1} \geq 6$. Note that in this case, $\left(P_{6}\right)_{i-1}=\left(P_{6}\right)_{i+3}$ and hence $x_{i-1}=x_{i+3}$.

Let $n \geq 5$. If $x_{i}=1, x_{i+1}=1, x_{i+2}=2$, and $x_{i+3}=0$ then $x_{i+4}=4$. Also, $x_{i+5} \leq 3$; otherwise $D^{\prime}=D \backslash\left[\left(P_{6}\right)_{i+4} \cup\left(P_{6}\right)_{i+5}\right] \cup$ $\{(0, i+4),(1, i+6),(2, i+3),(2, i+5),(4, i+4),(4, i+6),(5, i+$ $4)\}$ will be a dominating set with $\left|D^{\prime}\right|<|D|$, a contradiction. Clearly, $(0, i+1),(5, i+1) \notin D$. Also $(1, i+1),(4, i+1) \notin D$, because if $(1, i+1) \in D$, then $(0, i+2) \in D$ or $(1, i+2) \in D$, each of which will be a contradiction, since $(3, i+1),(4, i+1)$ and $(5, i+1)$ cannot be dominated by one vertex from $\left(P_{6}\right)_{i}$ and one vertex from $\left(P_{6}\right)_{i+2}$. Similar contradiction will be arrived for $(4, i+1) \in D$. Let $(2, i+1) \in D$. Then $(0, i+2),(4, i+2) \in D$. Hence we have $(1, i+4),(2, i+4),(3, i+4),(5, i+4) \in D$. Therefore we can construct $D^{\prime}=[D \backslash\{(2, i+4),(3, i+4)\}] \cup$ $\{(2, i+3),(3, i+5)\}$. In a similar way we can construct $D^{\prime}$ when $(3, i+2) \in D$. Repeating this construction for all $i$ for 
which $x_{i}=x_{i+1}=1, x_{i+2}=2, x_{i+3}=0$, and $x_{i-1}=0, x_{i}=2$, $x_{i+1}=1, x_{i+2}=1$, we get a minimum dominating set with the desired property.

Lemma 18. Consider a minimum dominating set $D$ of $P_{6} \square C_{n}$ with the property as stated in Lemma 17. Let $x_{i}=\left|\left(P_{6}\right)_{i} \cap D\right|, i=$ $0,1, \ldots, n-1$.

Then $\left(x_{i} / 2\right)+x_{i+1}+x_{i+2}+\left(\left(x_{i+3}\right) / 2\right) \geq 4$.

Proof. Case 1. Consider $x_{i+1}=0$; this implies $x_{i+2} \geq 2$ (since $\left.x_{i} \leq 4\right)$.

Subcase 1.1. Consider $x_{i+2}=2$; this implies $x_{i}=4$.

Subcase 1.2. Consider $x_{i+2}=3$; this implies $x_{i} \geq 3$.

Subcase 1.3. Consider $x_{i+2}=4$.

For every subcase we get the desired result.

Case 2. Consider $x_{i+1}=1$; this implies $x_{i+2} \geq 1$.

Subcase 2.1. Consider $x_{i+2}=1$; this implies $x_{i} \geq 2, x_{i+3} \geq 2$.

Subcase 2.2. Consider $x_{i+2}=2$; this implies $x_{i} \geq 1$ which implies $x_{i}+x_{i+3} \geq 2$ (since if $x_{i}=1$ then by Lemma $17 x_{i+3} \geq$ $1)$.

Subcase 2.3. Consider $x_{i+2} \geq 3$.

For every subcase we get the desired result.

Case 3. Consider $x_{i+1}=2$.

Subcase 3.1. Consider $x_{i+2}=0$; this implies $x_{i+3} \geq 4$.

Subcase 3.2. Consider $x_{i+2}=1$; this implies $x_{i+3} \geq 1$ which implies $x_{i}+x_{i+3} \geq 2$ (by Lemma 17).

Subcase 3.3. Consider $x_{i+2} \geq 2$.

For every subcase we get the desired result.

Case 4. Consider $x_{i+1}=3$.

Subcase 4.1. Consider $x_{i+2}=0$; this implies $x_{i+3} \geq 3$ as in Subcase 1.2.

Subcase 4.2. Consider $x_{i+2} \geq 1$.

For every subcase we get the desired result.

Case 5. Consider $x_{i+1}=4$.

In this case also $\left(x_{i} / 2\right)+x_{i+1}+x_{i+2}+\left(x_{i+3} / 2\right) \geq 4$.

Based on Lemma 17, we prove the following theorem which provides a lower bound on $\gamma\left(P_{6} \square C_{n}\right)$, for $n \geq 3$.

Theorem 19. For $n \geq 3, \gamma\left(P_{6} \square C_{n}\right) \geq 4 n / 3$.

Proof. Case 1 . When $n=3$, for any dominating set $D,|D| \geq$ $18 / 5>3$ (since the total number of vertices is $6 \times 3=18$ and one vertex of $D$ can dominate at most 5 vertices including itself). Therefore $|D| \geq 4=((4 \times 3) / 3)$. Hence the theorem is true for $n=3$.
Case 2. For $n \geq 4$ consider a minimum dominating set $D$ of $P_{6} \square C_{n}$ with the property as stated in Lemma 17. Then $\left(x_{i} / 2\right)+x_{i+1}+x_{i+2}+\left(x_{i+3} / 2\right) \geq 4 \forall i=0,1, \ldots, n-1$. Therefore $\sum_{i=0}^{n-1}\left\{\left(x_{i} / 2\right)+x_{i+1}+x_{i+2}+\left(x_{i+3} / 2\right)\right\} \geq 4 n$ that implies $3 \sum_{i=0}^{n-1} x_{i} \geq 4 n$ that implies $\sum_{i=0}^{n-1} x_{i} \geq 4 n / 3$; that is, $|D| \geq 4 n / 3$, hence the theorem.

Remark 20. For simplification, henceforth, in all the figures, we are only considering the grid structure avoiding the circular arks to represent the circular grid as shown in Figure 5.

The following theorem provides domination numbers and dominating sets for some particular cyclic grid graphs which will help in providing the upper bounds for $\gamma\left(P_{6} \square C_{n}\right)$, for $n>7$ as addressed in Theorem 23 .

\section{Theorem 21. Consider the following}

(a) $\gamma\left(P_{6} \square C_{3}\right)=5$, (b) $\gamma\left(P_{6} \square C_{4}\right)=6$, (c) $\gamma\left(P_{6} \square C_{5}\right)=8$, (d) $\gamma\left(P_{6} \square C_{6}\right)=9$, and (e) $\gamma\left(P_{6} \square C_{7}\right)=11$.

Proof. (a) A dominating set for $P_{6} \square C_{3}$ is $D_{6,3}=\{(0,1),(2,0)$, $(2,2),(4,1),(5,1)\}$, as shown in Figure 6. As $\left|D_{6,3}\right|=5$, $\gamma\left(P_{6} \square C_{3}\right) \leq 5$. We want to show $\gamma\left(P_{6} \square C_{3}\right)=5$.

If possible let there exist a dominating set $D$, of $P_{6} \square C_{3}$ with $|D| \leq 4$.

Let $x_{i}=\left|\left(C_{3}\right)_{i} \cap D\right|, \quad i=0,1,2,3,4,5$.

Therefore,

(i) $x_{0}+x_{1}+\cdots+x_{5} \leq 4$,

(ii) $3 x_{0}+x_{1} \geq 3$,

(iii) $x_{0}+3 x_{1}+x_{2} \geq 3$,

(iv) $x_{1}+3 x_{2}+x_{3} \geq 3$,

(v) $x_{2}+3 x_{3}+x_{4} \geq 3$,

(vi) $x_{3}+3 x_{4}+x_{5} \geq 3$,

(vii) $x_{4}+3 x_{5} \geq 3$.

We claim that $x_{0} \geq 1$; otherwise, if possible let $x_{0}=0$; then $x_{1} \geq 3$.

Therefore, $x_{2}+x_{3}+x_{4}+x_{5} \leq 1$.

This implies $x_{4}+x_{5} \leq 1$ and hence $x_{5} \geq 1$ (from (vii)).

Therefore, $x_{5}=1$ and then $x_{1}+x_{5} \geq 4$ implies $x_{0}=x_{2}=$ $x_{3}=x_{4}=0$, contradicting $(\mathrm{v})$, hence the claim.

Similarly, we can show that $x_{5} \geq 1$.

Therefore, $x_{1}+x_{2}+x_{3}+x_{4} \leq 2 \Rightarrow x_{3} \geq 1, x_{2} \geq 1$ (using (iv) and (v)).

Hence, $x_{0}=x_{2}=x_{3}=x_{5}=1$ which implies $x_{1}=x_{4}=0$, contradicting (iii).

Therefore, $\gamma\left(P_{6} \square C_{3}\right)=5$.

(b) A dominating set for $P_{6} \square C_{4}$ is $D_{6,4}=\{(0,3),(1,1)$, $(2,3),(3,0),(4,2),(5,0)\}$, as shown in Figure 6 . As $\left|D_{6,4}\right|=6$, $\gamma\left(P_{6} \square C_{4}\right) \leq 6$. We want to show $\gamma\left(P_{6} \square C_{4}\right)=6$.

If possible let there exist a dominating set $D$ of $P_{6} \square C_{4}$ with $|D| \leq 5$.

Let $x_{i}=\left|\left(C_{4}\right)_{i} \cap D\right|, \quad i=0,1,2,3,4,5$.

Therefore,

(i) $x_{0}+x_{1}+\cdots+x_{5} \leq 5$, 


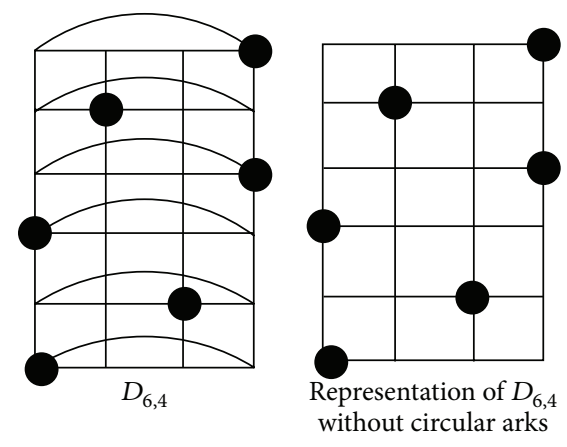

Figure 5: Dominating set for $P_{6} \square C_{4}$ and its representation in grid form without circular arks.
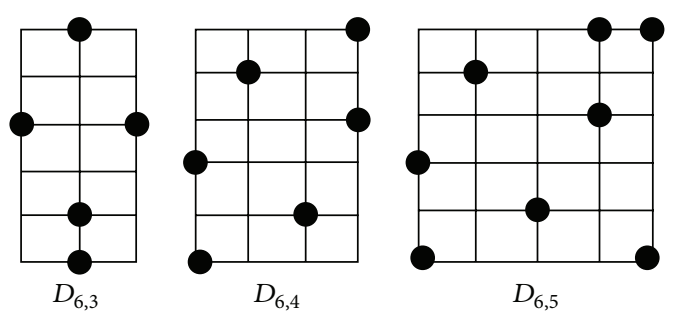

Figure 6: Dominating sets for $P_{6} \square C_{3}, P_{6} \square C_{4}$, and $P_{6} \square C_{5}$.
(ii) $3 x_{0}+x_{1} \geq 4$,
(iii) $x_{0}+3 x_{1}+x_{2} \geq 4$,
(iv) $x_{1}+3 x_{2}+x_{3} \geq 4$,
(v) $x_{2}+3 x_{3}+x_{4} \geq 4$,
(vi) $x_{3}+3 x_{4}+x_{5} \geq 4$,
(vii) $x_{4}+3 x_{5} \geq 4$.

We claim that $x_{0} \geq 1$; otherwise, if possible let $x_{0}=0$. Then $x_{1} \geq 4$, which implies $x_{2}+x_{3}+x_{4}+x_{5} \leq 1$, contradicting (vii), hence the claim.

Therefore, $x_{0} \geq 1$. Similarly, we can show that $x_{5} \geq 1$. Next we claim that $x_{1} \geq 1$. If possible let $x_{1}=0$. Therefore, $x_{0}+x_{2} \geq 4 \Longrightarrow x_{3}+x_{4}+x_{5} \leq 1$, contradicting (vii), hence the claim. Therefore $x_{1} \geq 1$. Similarly, $x_{5}, x_{3}, x_{4} \geq 1$, contradicting (i).

Therefore, $\gamma\left(P_{6} \square C_{4}\right)=6$.

(c) A dominating set for $P_{6} \square C_{5}$ is $D_{6,5}=\{(0,3)$, $(0,4),(1,1),(2,3),(3,0),(4,2),(5,0),(5,4)\}$ as shown in Figure 6.

8.

As $\left|D_{6,5}\right|=8, \gamma\left(P_{6} \square C_{5}\right) \leq 8$. We want to show $\gamma\left(P_{6} \square C_{5}\right)=$

If possible let there exist a dominating set $D$ of $P_{6} \square C_{5}$ with $|D| \leq 7$.

Let $x_{i}=\left|\left(C_{4}\right)_{i} \cap D\right|, \quad i=0,1,2,3,4,5$.

Therefore,

(i) $x_{0}+x_{1}+\cdots+x_{5} \leq 7$,

(ii) $3 x_{0}+x_{1} \geq 5$,

(iii) $x_{0}+3 x_{1}+x_{2} \geq 5$,

(iv) $x_{1}+3 x_{2}+x_{3} \geq 5$, (v) $x_{2}+3 x_{3}+x_{4} \geq 5$,

(vi) $x_{3}+3 x_{4}+x_{5} \geq 5$,

(vii) $x_{4}+3 x_{5} \geq 5$.

We claim that $x_{0} \geq 1$; otherwise, if possible let $x_{0}=0$. Then $x_{1} \geq 5$, which implies $x_{2}+x_{3}+x_{4}+x_{5} \leq 2$.

From (vii) we get, $x_{5}=2$ and $x_{4}=0$; hence, $x_{2}=x_{3}=0$, a contradiction.

Similarly, we can show that $x_{5} \geq 1$. Now we claim that $x_{1} \geq 1$; otherwise, $x_{0}+x_{2} \geq 5$, which implies $x_{3}+x_{4}+x_{5} \leq 2$. Therefore, from (vii), $x_{5}=2$ and $x_{4}=0=x_{3}$, contradicting (vi). Similarly, $x_{4}, x_{2}, x_{3} \geq 1$. Next we claim that $x_{0} \geq 2$; otherwise, $x_{0}=1$ and $x_{1} \geq 2$. Therefore, $x_{0}=x_{2}=x_{3}=$ $x_{4}=x_{5}=1$ and $x_{1}=2$, contradicting (vii). Similarly, $x_{5} \geq 2$, contradicting (i).

(d) A dominating set for $P_{6} \square C_{6}$ is $D_{6,6}=\{(0,0),(0,4)$, $(1,2),(2,5),(3,1),(3,3),(4,0),(5,2),(5,4)\}$ as shown in Figure 7. As $\left|D_{6,6}\right|=9, \gamma\left(P_{6} \square C_{6}\right) \leq 9$. We want to show $\gamma\left(P_{6} \square C_{6}\right)=9$.

If possible let there exist a dominating set $D$ of $P_{6} \square C_{6}$ with $|D| \leq 8$.

Let $x_{i}=\left|\left(C_{4}\right)_{i} \cap D\right|, \quad i=0,1,2,3,4,5$.

Therefore,

(i) $x_{0}+x_{1}+\cdots+x_{5} \leq 8$,

(ii) $3 x_{0}+x_{1} \geq 6$,

(iii) $x_{0}+3 x_{1}+x_{2} \geq 6$,

(iv) $x_{1}+3 x_{2}+x_{3} \geq 6$,

(v) $x_{2}+3 x_{3}+x_{4} \geq 6$,

(vi) $x_{3}+3 x_{4}+x_{5} \geq 6$,

(vii) $x_{4}+3 x_{5} \geq 6$.

We claim that $x_{0} \geq 1$; otherwise, if possible let $x_{0}=0$. Then $x_{1} \geq 6$, which implies $x_{4}+x_{5} \leq 2$ and hence $x_{5}=2$ (by (vii)).

Hence, $x_{0}=x_{2}=x_{3}=x_{4}=0$, a contradiction.

Therefore, $x_{0} \geq 1$. Similarly we can show that $x_{5} \geq 1$.

Now we claim that $x_{1} \geq 1$; otherwise, $x_{0}+x_{2} \geq 6$, which implies that $x_{4}+x_{5} \leq 2$. Therefore, $x_{5}=2$ (by (vii)) and hence $x_{1}=x_{3}=x_{4}=0$, contradicting (vi). Hence, $x_{1} \geq 1$. Similarly $x_{2}, x_{3}, x_{4} \geq 1$.

Next we claim that $x_{0} \geq 2$; otherwise, let $x_{0}=1$. Then $x_{1} \geq 3$.

Therefore, $x_{0}=x_{2}=x_{3}=x_{4}=x_{5}=1$ and hence $x_{1}=3$ which contradicts (vii).

Hence, $x_{0} \geq 2$. Similarly, $x_{5} \geq 2$. Therefore, $x_{0}=x_{5}=2$ and $x_{1}=x_{2}=x_{3}=x_{4}=1$, which contradicts (iv). Hence, $\gamma\left(P_{6} \square C_{6}\right)=9$.

(e) A dominating set for $P_{6} \square C_{7}$ is $D_{6,7}=\{(0,0),(0,4)$, $(1,2),(2,5),(2,6),(3,1),(3,3),(4,0),(4,6),(5,2),(5,4)\}$ as shown in Figure 7. As $\left|D_{6,7}\right|=11, \gamma\left(P_{6} \square C_{7}\right) \leq 11$. We want to show $\gamma\left(P_{6} \square C_{7}\right)=11$.

If possible let there exist a dominating set $D$ of $P_{6} \square C_{7}$ with $|D| \leq 10$.

Let $x_{i}=\left|\left(C_{4}\right)_{i} \cap D\right|, i=0,1,2,3,4,5$.

Therefore,

(i) $x_{0}+x_{1}+\cdots+x_{5} \leq 10$, 

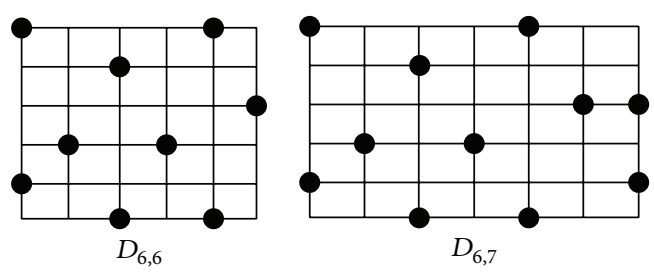

Figure 7: Dominating sets for $P_{6} \square C_{6}$ and $P_{6} \square C_{7}$.

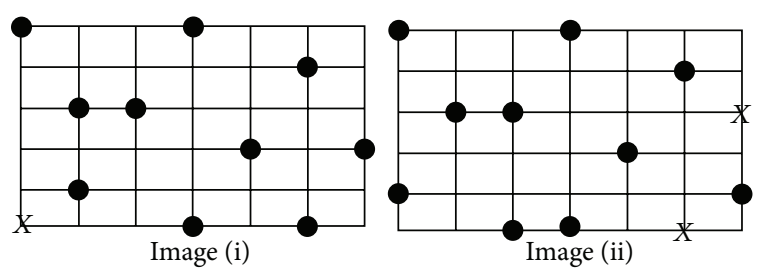

FIGURE 8: Figures show nonpossibility of dominating sets in some cases.

(ii) $3 x_{0}+x_{1} \geq 7$,

(iii) $x_{0}+3 x_{1}+x_{2} \geq 7$

(iv) $x_{1}+3 x_{2}+x_{3} \geq 7$

(v) $x_{2}+3 x_{3}+x_{4} \geq 7$

(vi) $x_{3}+3 x_{4}+x_{5} \geq 7$

(vii) $x_{4}+3 x_{5} \geq 7$.

By similar arguments, as in (d) we can prove, $x_{0}, x_{5} \geq 2$ and $x_{1}, x_{2}, x_{3}, x_{4} \geq 1$.

We claim that $x_{1} \leq 2$. If possible let $x_{1} \geq 3$. Then $x_{0}=$ $x_{5}=2, x_{1}=3$, and $x_{2}=x_{3}=x_{4}=1$, contradicting $(\mathrm{v})$, which implies that $x_{1} \leq 2$. Similarly, $x_{2}, x_{3}, x_{4} \leq 2$.

Next we claim that $x_{0} \leq 2$; otherwise, let $x_{0} \geq 3$. Now clearly $x_{0} \leq 3$ and hence $x_{0}=3$.

Hence, we have the following cases: $\left(x_{1}, x_{2}, x_{3}, x_{4}, x_{5}\right)=$ $(2,1,1,1,2)$ or $(1,2,1,1,2)$ or $(1,1,2,1,2)$ or $(1,1,1,2,2)$ or $(1,1,1,1,3)$ and each of the cases lead to contradictions.

Therefore, $x_{0}=2$. Similarly, we can show that $x_{5}=2$.

Hence the possibilities are $\left(x_{1}, x_{2}, x_{3}, x_{4}\right)=(1,1,2,2)$ or $(2,2,1,1)$ or $(2,1,1,2)$ or $(1,2,2,1)$ or $(2,1,2,1)$ or $(1,2,1,2)$.

It is easy to show that first three cases lead to contradictions.

Hence the possibilities become $\left(x_{0}, x_{1}, x_{2}, x_{3}, x_{4}, x_{5}\right)=$ $(2,1,2,2,1,2)$ or $(2,2,1,2,1,2)$ or $(2,1,2,1,2,2)$.

If possible let there exist a dominating set $D$ with $\left(x_{0}, x_{1}, x_{2}, x_{3}, x_{4}, x_{5}\right)=(2,1,2,2,1,2)$.

Without loss of generality, let $(0,0) \in D$.

Therefore, $(0,1),(0,2),(0,5),(0,6) \notin D$.

Without loss of generality, let $(0,3) \in D$. Therefore $(1,5) \in D$. This implies $(2,1),(2,2) \in D$.

Hence, $(3,4),(3,6) \in D$; then, $(4,0),(4,3),(4,4),(4,5)$, $(4,6) \notin D$.

Now if $(4,1) \in D$, then, $(5,3),(5,5), \in D$. But then $(5,0)$ is not dominated by any vertex of $D$ as shown in Figure 8 .

And if $(4,2) \in D$, then, $(5,0),(5,5), \in D$. But then $(5,3)$ is not dominated by any vertex of $D$.
Hence there does not exist any dominating set $D$ with $\left(x_{0}, x_{1}, x_{2}, x_{3}, x_{4}, x_{5}\right)=(2,1,2,2,1,2)$.

By similar manner we can show there does not exist any dominating set with $\left(x_{0}, x_{1}, x_{2}, x_{3}, x_{4}, x_{5}\right)=(2,1,2,1,2,2)$, as shown in Figure 8.

Again there does not exist any dominating set $D$ with $\left(x_{0}, x_{1}, x_{2}, x_{3}, x_{4}, x_{5}\right)=(2,2,1,2,1,2)$ as this case is similar as the above case. Hence, $\gamma\left(P_{6} \square C_{7}\right)=11$.

The following theorem provides bounds for dominating sets that will help in proving the main Theorem 23.

Theorem 22. Consider the following:

(i) $\gamma\left(P_{6} \square C_{8}\right) \leq 12$, (ii) $\gamma\left(P_{6} \square C_{9}\right) \leq 14$, (iii) $\gamma\left(P_{6} \square C_{10}\right) \leq$ 15, (iv) $\gamma\left(P_{6} \square C_{11}\right) \leq 16$, (v) $\gamma\left(P_{6} \square C_{12}\right) \leq 18$, (vi) $\gamma\left(P_{6} \square C_{13}\right)$ $\leq 20$, (vii) $\gamma\left(P_{6} \square C_{14}\right) \leq 20$, (viii) $\gamma\left(P_{6} \square C_{15}\right) \leq 23$, (ix) $\gamma\left(P_{6} \square C_{16}\right) \leq 24$, (x) $\gamma\left(P_{6} \square C_{17}\right) \leq 26$, (xi) $\gamma\left(P_{6} \square C_{18}\right) \leq$ 26, (xii) $\gamma\left(P_{6} \square C_{19}\right) \leq 28$, (xiii) $\gamma\left(P_{6} \square C_{20}\right) \leq 30$, (xiv) $\gamma\left(P_{6} \square C_{21}\right) \leq 31,(x v) \gamma\left(P_{6} \square C_{22}\right) \leq 31$, (xvi) $\gamma\left(P_{6} \square C_{23}\right)$ $\leq 34,(x v i i) \gamma\left(P_{6} \square C_{24}\right) \leq 35$, (xviii) $\gamma\left(P_{6} \square C_{25}\right) \leq 36$, (xix) $\gamma\left(P_{6} \square C_{26}\right) \leq 38$, and $(x x) \gamma\left(P_{6} \square C_{27}\right) \leq 40$.

Proof. Dominating sets for each of the cases are shown in Figures 12, 13, 14, 15, 16, 17, and 18.

Theorem 23. For $n>7$,

$$
\begin{aligned}
& \gamma\left(P_{6} \square C_{n}\right) \\
& \quad \leq \begin{cases}\left\lceil\frac{10 n}{7}\right\rceil, & \text { for } n \equiv 0,4,5,8,10,11,12(\bmod 14) ; \\
\left\lceil\frac{10 n}{7}\right\rceil+1, & \text { otherwise. }\end{cases}
\end{aligned}
$$

Proof. For $3 \leq n \leq 27$, the above inequalities have already been proved in Theorems 21 and 22. For $n \geq 28$ one can easily find a dominating set for $\gamma\left(P_{6} \square C_{n}\right)$ using repeated modified concatenation between the dominating set for $\gamma\left(P_{6} \square C_{14}\right)$ with dominating set for $\gamma\left(P_{6} \square C_{n-14}\right)$.

The following theorem provides a lower bound for $\gamma\left(P_{6} \square C_{n}\right)$, for $n>7$.

Theorem 24. For $n>7$, if there exists a minimum dominating set $D$ with $\left|\left(P_{6}\right)_{i} \cap D\right|=1$ or 2 for all $i$, then $\gamma\left(P_{6} \square C_{n}\right) \geq\lceil 10 n / 7\rceil$ resulting $\gamma\left(P_{6} \square C_{n}\right)=\lceil 10 n / 7\rceil+0$ or 1 , accordingly.

Proof. It is enough to show that, for a dominating set $D$ with $\left|\left(P_{6}\right)_{i} \cap D\right|=1$ or 2 for all $i$, it holds that $|D| \geq\lceil 10 n / 7\rceil$.

We say the configuration of the type $k_{1} k_{2} k_{3} \ldots$ occurs in a dominating set $D$, where $k_{1}, k_{2}, k_{3}, \ldots$ are 1 or 2 , if there are consecutive columns in the graph with $k_{1}, k_{2}, k_{3} \ldots$ many common vertex (or vertices) with $D$.

We have the following observations which can be verified considering all possible cases (we omit the verification because it has several cases and subcases and require similar types of arguments).

(1) There cannot be three consecutive columns with only one vertex common with $D$; that is, the configuration 

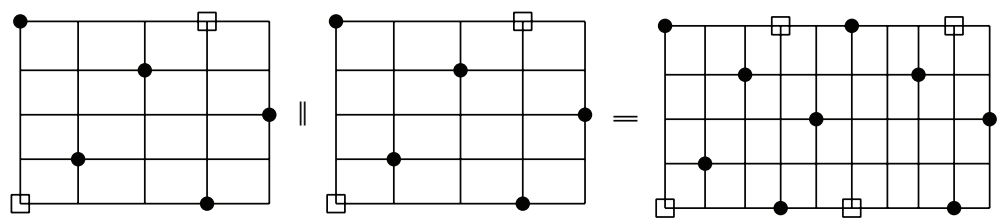

FIGURE 9: Modified concatenation of subsets of dominating sets columnwise, where $\square$ denotes the vertex not captured by the subset.

of the type 111 cannot occur in $D$. This is clear from Remark 4.

(2) If the configuration of the type 2112112 occurs, then just before and after these seven columns the configuration of the type 22 should occur; that is, the configuration of the type 22211211222 should occur where the previous configuration occurs inside this.

(3) If there are two configurations of the type 2112 which occurs and between these two configurations only 212 type configuration occurs then the configuration of the type 212 occurs at least twice.

(4) The configuration of the type 211221122112 cannot occur.

Now let there be $2 x+y$ many configurations of the type 2112 occur where $2 x$ many configurations occur in $x$ many pair and $y$ many occur without pairing. Also let $z$ many configurations of the type 22 occur and $t$ many configuration of the type 212 occur.

Then, $z \geq x$ and $t \geq 2(x+y-z)$. Now the number of columns which have one vertex in common with $D$ is $2(2 x+$ $y)+t$ and the number of columns which have two vertices in common with $D$ is $2 x+y+z+t$.

Therefore, $n=2(2 x+y)+t+(2 x+y+z+t)$ and $|D|=$ $2(2 x+y)+t+2(2 x+y+z+t)$.

Hence, $7|D|-10 n=4(2 x+y+z+t)-3(2(2 x+y)+t)=$ $-4 x-2 y+t+4 z=2(z-x)+t-2(x+y-z) \geq 0$. So $|D| \geq 10 n / 7$.

Remark 25. For $n \geq 3, \gamma\left(P_{6} \square C_{n}\right)>\gamma\left(P_{6}\right) \cdot \gamma\left(C_{n}\right)=2 \cdot\lceil n / 3\rceil$.

\section{Bounds for General Case}

In this section we give rough bounds of $\gamma\left(P_{m} \square C_{n}\right)$ for any $m, n \geq 5$. If $m=5 p+k$ and $n=5 q+l$, where $p, q$ are natural numbers and $k, l=0,1,2,3,4$, a lower bound for $\gamma\left(P_{m} \square C_{n}\right)$ is $\lceil m n / 5\rceil=5 p q+k q+p l+\lceil k l / 5\rceil$, as a vertex can dominate at most 5 vertices. An upper bound of $\gamma\left(P_{m} \square C_{n}\right)$ is given by $5 p q+(k+2) q+(l+1) p$ if $l \neq 0$ and $5 p q+(k+2) q$ if $l=0$. A dominating set with above cardinality can be constructed as follows.

Step 1. Consider the subset $D=\{(0,0),(1,2),(2,4),(3,1)$, $(4,3)\}$ of $P_{5} \square C_{n}$ as shown in Figure 9. Modified concatenation columnwise and concatenation rowwise are shown in Figures 9 and 10 , respectively.

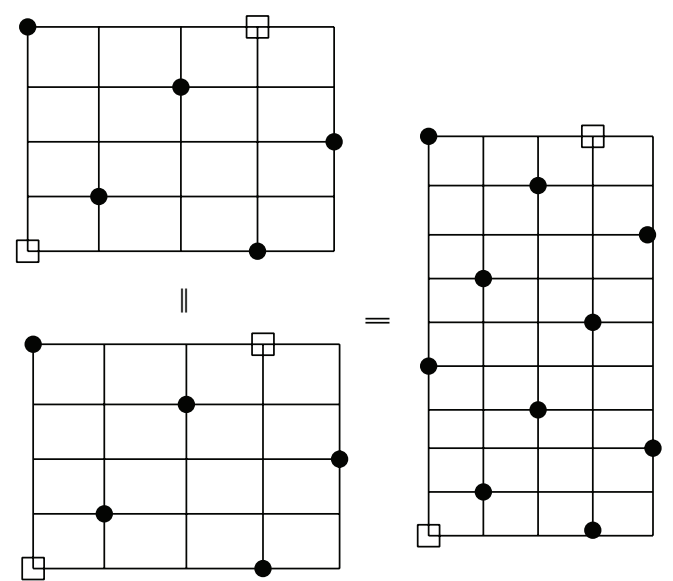

FIGURE 10: Concatenation of subsets of dominating sets row wise.

Step 2. Construct a subset of $P_{5 p} \square C_{5 q}$ using modified concatenation columnwise $q$ many times and using concatenation rowwise $p$ many times the set $D$ as shown in Figure 11 .

Step 3. Add extra $q$ suitably chosen vertices in the first row and extra $q$ suitably chosen vertices in the last row to construct dominating set for $P_{5 p} \square C_{5 q}$. Hence a dominating set of $P_{5 p} \square C_{5 q}$ is $\{(5 i, 5 j),(5 i+1,5 j+2),(5 i+2,5 j+4),(5 i+$ $3,5 j+1),(5 i+4,5 j+3): i=0,1, \ldots, p-1, j=0,1, \ldots, q-$ $1\} \cup\{(0,5 j+4): j=0,1, \ldots, q-1\} \cup\{(5 p-1,5 j): j=$ $0,1, \ldots, q-1\}$.

Step 4. One can construct a dominating set for $P_{5 p+k} \square C_{5 q+l}$, $(k, l) \neq(0,0)$, using concatenation and modified concatenation between the above dominating set for $P_{5 p} \square C_{5 q}$ and a suitably chosen subset $D^{\prime}$ of vertices for extra $k$ rows and $l$ columns. The cardinality of $D^{\prime}$ will be $k q+(l+1) p$ if $l \neq 0$ and $k q$ if $l=0$.

Remark 26. We have already seen that if $m=5 p+k$ and $n=$ $5 q+l$, where $p, q$ are natural numbers and $k, l=0,1,2,3,4$, then

$$
\begin{aligned}
\left\lceil\frac{m n}{5}\right\rceil & =5 p q+k q+p l+\left\lceil\frac{k l}{5}\right\rceil \leq \gamma\left(P_{m} \square C_{n}\right) \\
& \leq \begin{cases}5 p q+(k+2) q+(l+1) p, & \text { if } l \neq 0 \\
5 p q+(k+2) q, & \text { if } l=0 .\end{cases}
\end{aligned}
$$



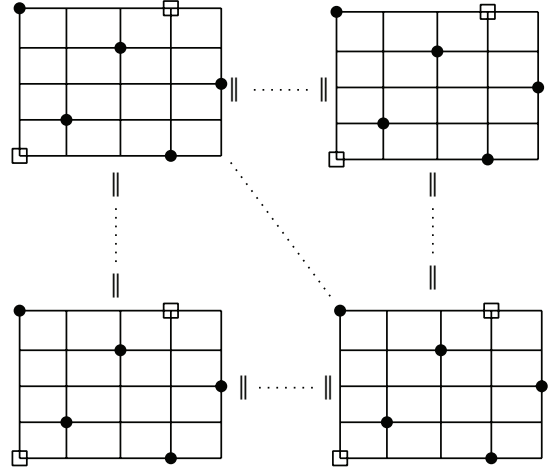

FIGURE 11: Modified concatenation and concatenation of subsets of dominating sets both column- and row wise.
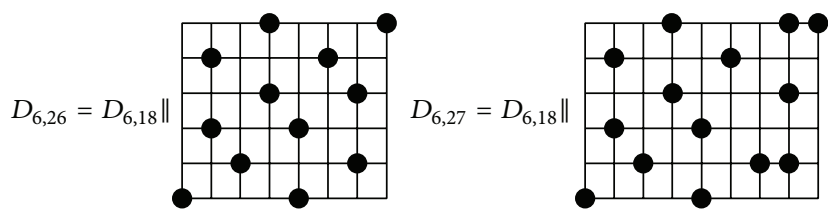

FIgURE 12: Dominating sets for $P_{6} \square C_{26}$ and $P_{6} \square C_{27}$.
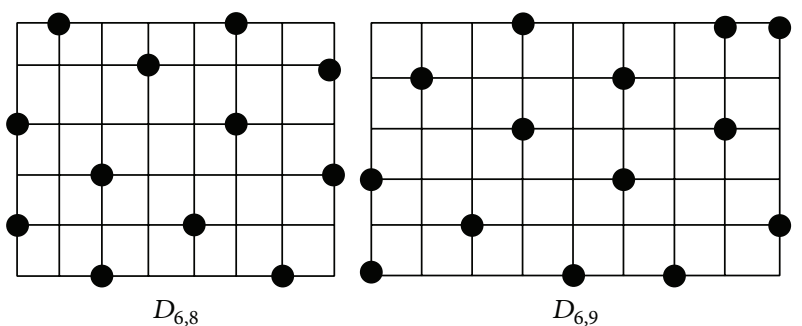

Figure 13: Dominating sets for $P_{6} \square C_{8}$ and $P_{6} \square C_{9}$.

Thus we have

$$
\begin{gathered}
1+\frac{l}{5 q} \leq \lim _{p \rightarrow \infty} \frac{\gamma\left(P_{m} C_{n}\right)}{5 p q} \leq 1+\frac{l+1}{5 q}, \quad \text { for } l \neq 0, \\
\lim _{p \rightarrow \infty} \frac{\gamma\left(P_{m} \square C_{n}\right)}{5 p q}=1, \quad \text { for } l=0 .
\end{gathered}
$$

Therefore, $1+(k / 5 p) \leq \lim _{q \rightarrow \infty}\left(\gamma\left(P_{m} \square C_{n}\right) / 5 p q\right) \leq 1+$ $((k+2) / 5 p)$, for $l=0$ and $\lim _{p, q \rightarrow \infty}\left(\gamma\left(P_{m} \square C_{n}\right) / 5 p q\right)=1$.

\section{Application of Domination Number in Wireless Sensor Networks}

Wireless sensor networks (WSN) provide a new communication and networking paradigms and myriad new applications. The wireless sensors have small size, low battery capacity, nonrenewable power supply, small processing power, limited
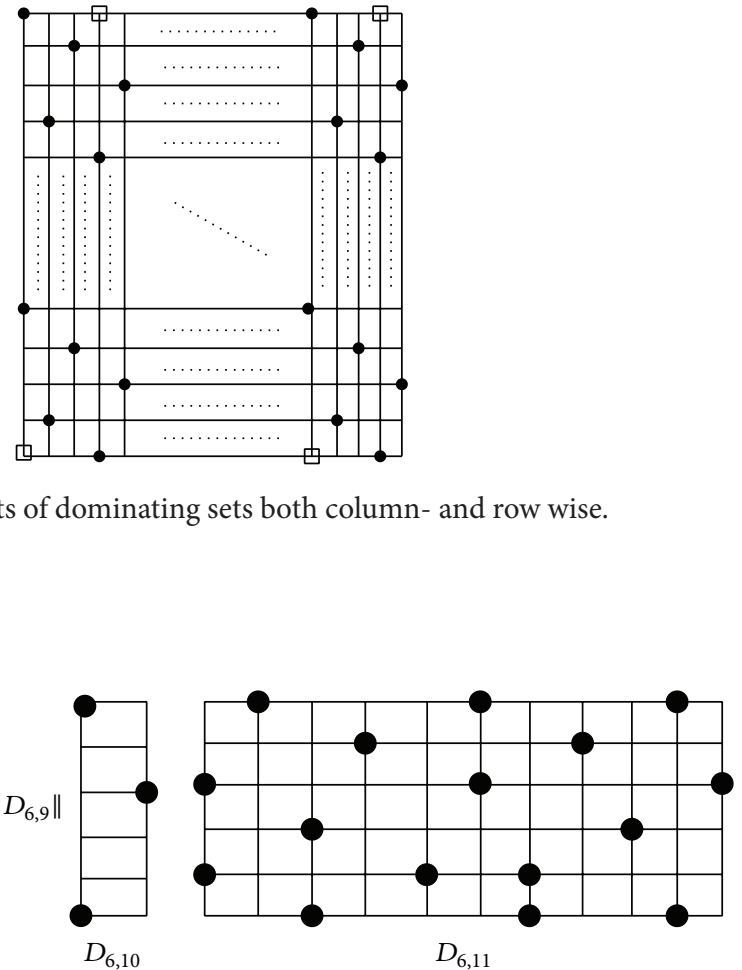

FIgURE 14: Dominating sets for $P_{6} \square C_{10}$ and $P_{6} \square C_{11}$.

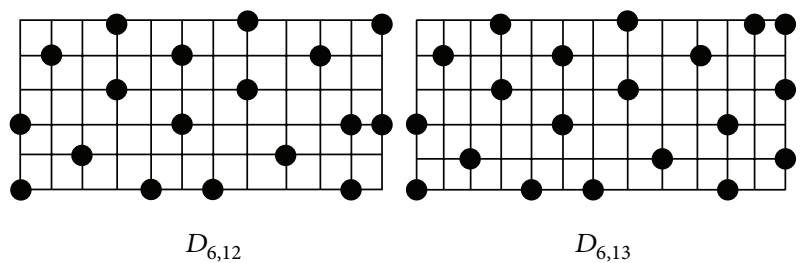

Figure 15: Dominating sets for $P_{6} \square C_{12}$ and $P_{6} \square C_{13}$.
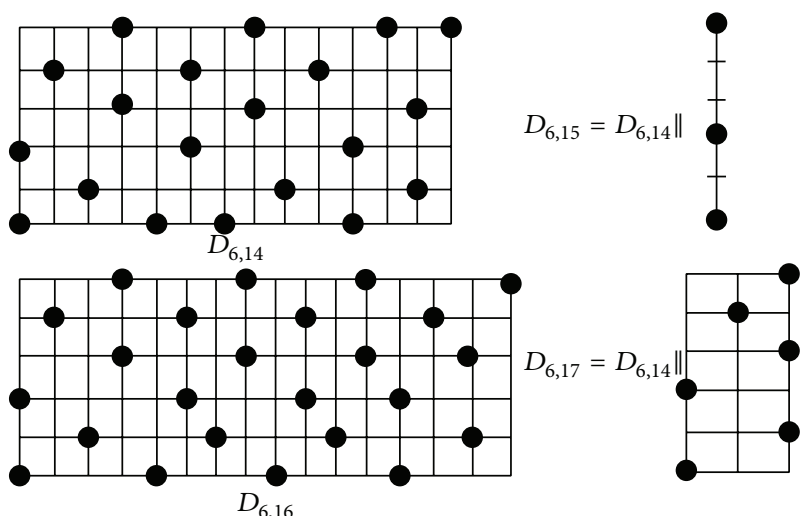

Figure 16: Dominating sets for $P_{6} \square C_{14}, P_{6} \square C_{15}, P_{6} \square C_{16}$, and $P_{6} \square C_{17}$.

buffer capacity, and low-power radio. They may measure distance, direction, speed, humidity, wind speed, soil makeup, temperature, chemicals, light and various other parameters. 


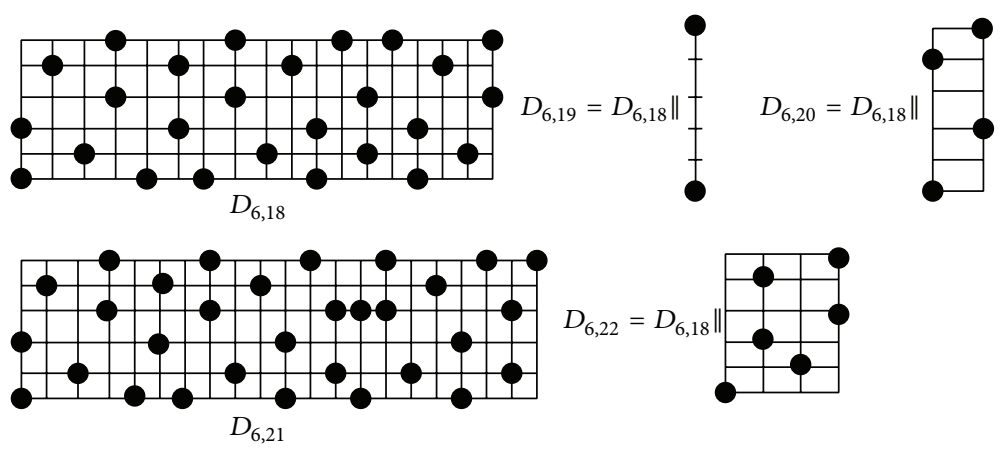

Figure 17: Dominating sets for $P_{6} \square C_{18}, P_{6} \square C_{19}, P_{6} \square C_{20}, P_{6} \square C_{21}$, and $P_{6} \square C_{22}$.

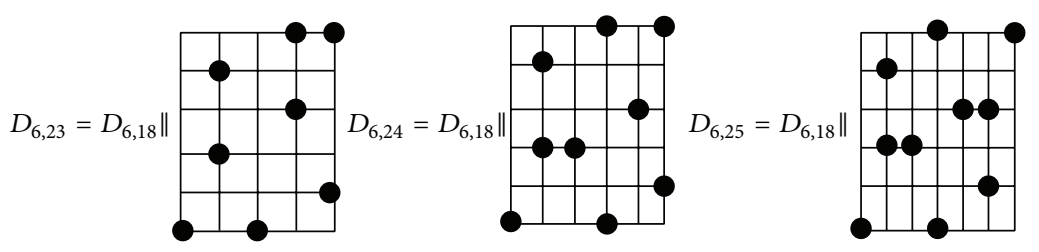

FIgURe 18: Dominating sets for $P_{6} \square C_{23}, P_{6} \square C_{24}$, and $P_{6} \square C_{25}$.

Recent advancements in wireless communications and electronics have enabled the development of low-cost WSN. A WSN usually consists of a large number of small sensor nodes, which are equipped with one or more sensors, some processing circuit and a wireless transceiver. One of the unique features of a WSN is random deployment in inaccessible terrains and cooperative effort that offers unprecedented opportunities for a broad spectrum of civilian and military applications; such as industrial automation, military tactical surveillance and national security [18]. Sensor Networks are useful in detecting topological events such as forest fires [19]. Sensor networks aim at monitoring their surroundings for event detection and object tracking [20]. Because of this surveillance goal, coverage is the functional basis of any sensor network. In order to fulfill its designated surveillance tasks, a sensor network must fully cover the Region of Interest (ROI) without leaving any internal sensing hole [21]. The ROI may be a rectangular grid, which may be divided into several squares.

In general, a sensor is placed at the center of a square, known as center node as shown in Figure 19. This sensor can detect events or tracking objects at the center node along with the four centers of the four adjacent squares which have a common edge with the center square. These four centers are known as Distance-one nodes as shown in Figure 19. The sensor placed at the center node cannot detect events or tracking objects placed at the center of the other squares, for example, Distance-two node as shown in Figure 19. Our objective is to place minimum number of sensors at the center of some selected squares in such a way that they can detect the events or tracking the objects at the center of all the squares. Then the minimum number of sensor required is the same as the domination number of the corresponding

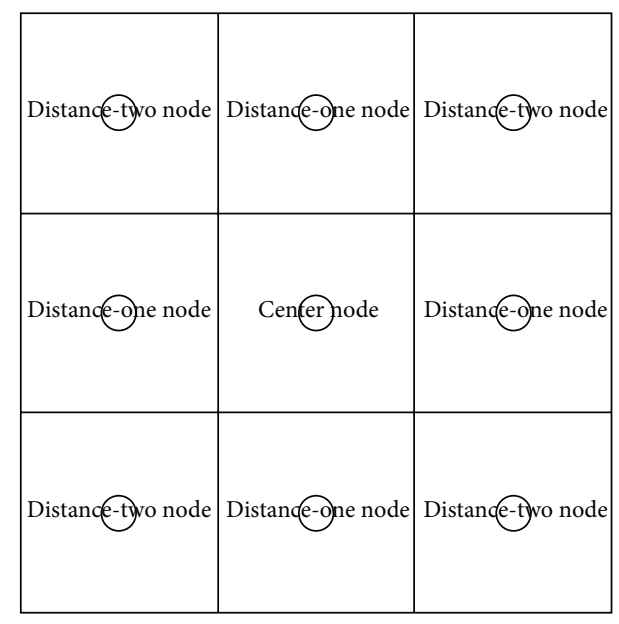

FIgURE 19: Nodes placed in cells of the ROI.

rectangular grid and a minimum dominating set will suggest which squares we have to choose.

\section{Conclusion}

In this paper we find the domination numbers of the graphs $P_{m} \square C_{n}, m=5, n \geq 3$. We also provide bounds on $\gamma\left(P_{6} \square C_{n}\right)$, $n \geq 3$. Minimum dominating sets corresponding to the above-mentioned graphs are also constructed. Moreover, we provide rough bounds on domination numbers of the graphs $\gamma\left(P_{m} \square C_{n}\right), m \geq 7$ and $n \geq 3$, and in future, we would like to provide sharper bounds. We also point out how domination 
numbers and minimum dominating sets may be useful to wireless sensor networks.

\section{Conflict of Interests}

The authors declare that there is no conflict of interests regarding the publication of this paper.

\section{References}

[1] T. Haynes, S. Hedetniemi, and P. Slater, Fundamentals of Domination in Graphs, Marcel Dekker, New York, NY, USA, 1998.

[2] J. E. Dunbar, S. T. Hedetniemi, M. A. Henning, and P. J. Slater, "Signed domination in graphs," in Graph Theory, Combinatorics and Applications, pp. 311-322, John Wiley \& Sons, 1995.

[3] O. Favaron, "Signed domination in regular graphs," Discrete Mathematics, vol. 158, no. 1-3, pp. 287-293, 1996.

[4] O. Morgenstern, "The colaboration between Oskar Morgenstern and John von Neumann on the theory of games," Journal of Economic Literature, vol. 14, pp. 805-816, 1976.

[5] S. T. Hedetniemi and R. C. Laskar, "Bibliography on domination in graphs and some basic definitions of domination parameters," Discrete Mathematics, vol. 86, no. 1-3, pp. 257-277, 1990.

[6] V. G. Vizing, "The cartesian product of graphs," Vychisl Sistemy, vol. 9, pp. 30-43, 1963.

[7] S. Gravier, "Total domination number of grid graphs," Discrete Applied Mathematics, vol. 121, no. 1-3, pp. 119-128, 2002.

[8] R. J. Faudree and R. H. Schelp, "The domination number for the product of graphs," Congressus Numerantium, vol. 79, pp. 29-33, 1990.

[9] M. El-Zahar and C. M. Pareek, "Dominationnumber of products of graphs," Ars Combinatoria, vol. 31, pp. 223-227, 1991.

[10] F. Harary, Graph Theory, Addison-Weslay, Reading, Mass, USA, 1969.

[11] S. Klavžar and N. Seifter, "Dominating cartesian products of cycles," Discrete Applied Mathematics, vol. 59, no. 2, pp. 129-136, 1995.

[12] B. N. Clark, C. J. Colbourn, and D. S. Johnson, "Unit disk graphs," Discrete Mathematics, vol. 86, no. 1-3, pp. 165-177, 1990.

[13] M. S. Jacobson and L. F. Kinch, "On the domination of the products of graphs I," Ars Combinatoria, vol. 18, pp. 33-44, 1983.

[14] T. Y. Chang and W. E. Clark, "The domination numbers of the $5 \times n$ and $6 \times n$ grid graphs," Journal of Graph Theory, vol. 17, pp. 81-107, 1993.

[15] M. H. El-Zahar, S. M. Khamis, and K. M. Nazzal, "On the domination number of the cartesian product of the cycle of length $n$ and any graph," Discrete Applied Mathematics, vol. 155, no. 4 , pp. 515-522, 2007.

[16] B. L. Hartnell and D. F. Rall, “On Vizing's conjecture," Congressus Numerantium, vol. 82, pp. 87-96, 1991.

[17] M. Nandi, S. Parui, and A. Adhikari, "Domination number of cylindrical grids," Applied Mathematics and Computation, vol. 217, no. 10, pp. 4879-4889, 2011.

[18] G. J. Pottie and W. J. Kaiser, "Wireless integrated network sensors," Communications of the ACM, vol. 43, no. 5, pp. 51-58, 2000.

[19] C. Farah, F. Schwaner, A. Abedi, and M. Worboys, "Distributed homology algorithm to detect topological events via wireless sensor networks," IET Wireless Sensor Systems, vol. 1, no. 3, pp. 151-160, 2011.

[20] F. Martincic and L. Schwiebert, "Introduction to wireless sensor networking," in Handbook of Sensor Networks: Algorithms and Architectures, I. Stojmenovic, Ed., chapter 1, John Wiley \& Sons, 2005.

[21] X. Bai, S. Kumar, D. Xuan, Z. Yun, and T. H. Lai, "Deploying wireless sensors to achieve both coverage and connectivity," in Proceedings of the 7th ACM International Symposium on Mobile Ad Hoc Networking and Computing (MOBIHOC '06), pp. 131142, May 2006. 


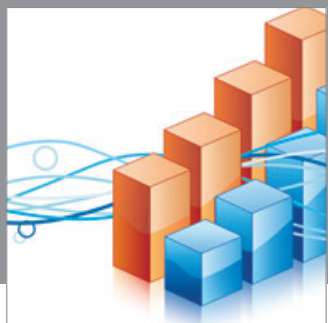

Advances in

Operations Research

mansans

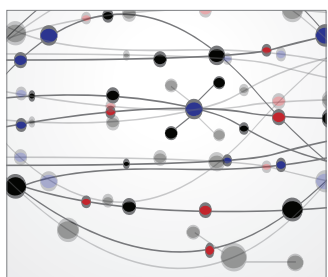

The Scientific World Journal
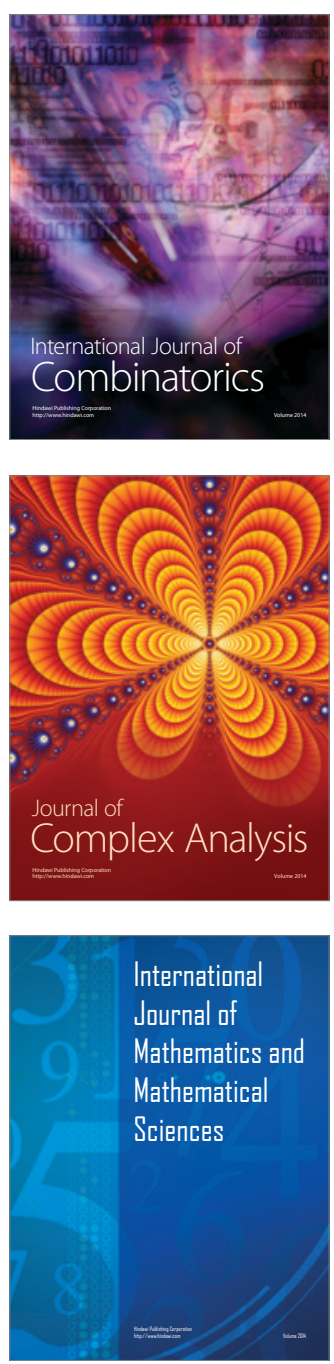
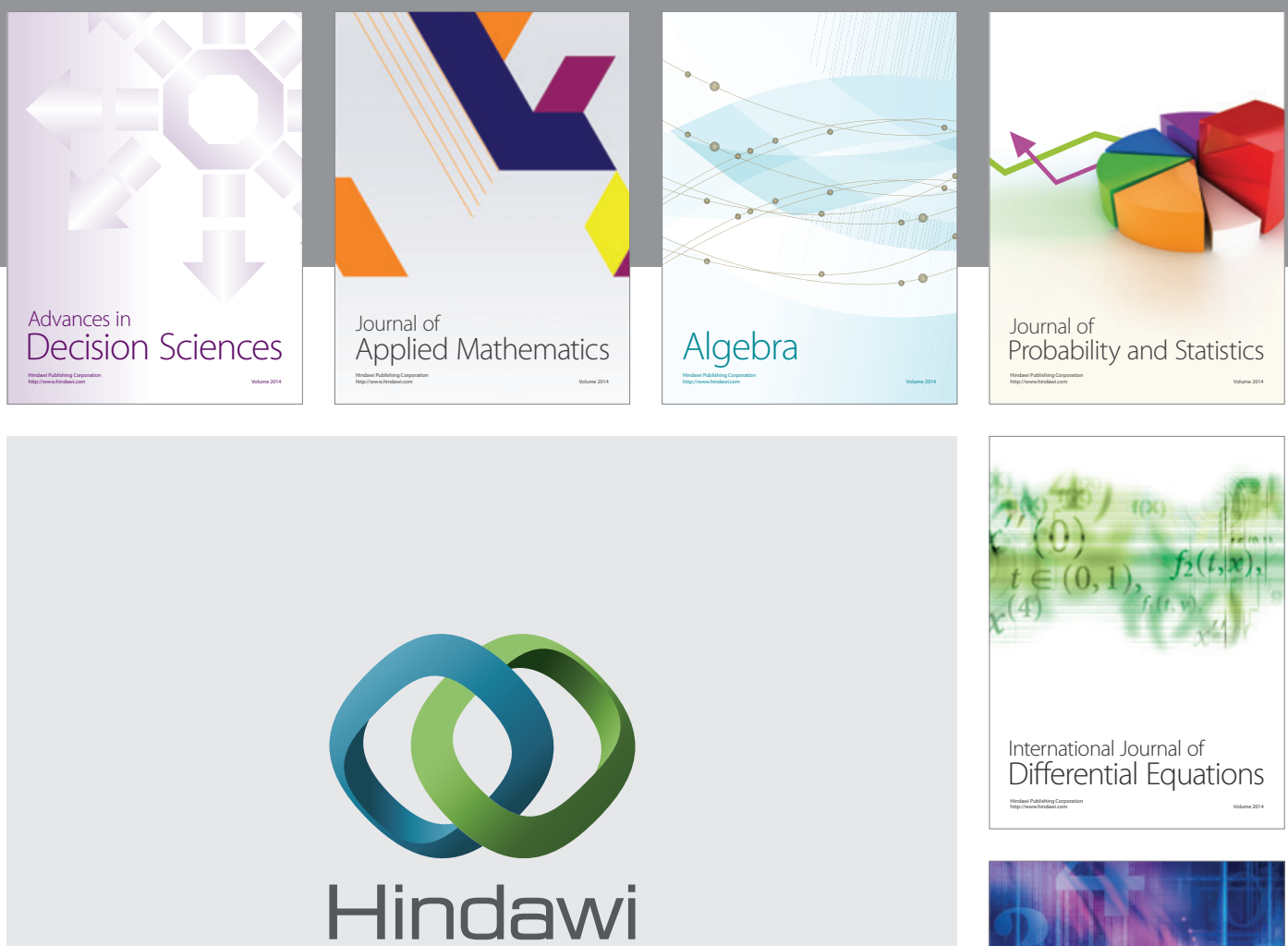

Submit your manuscripts at http://www.hindawi.com
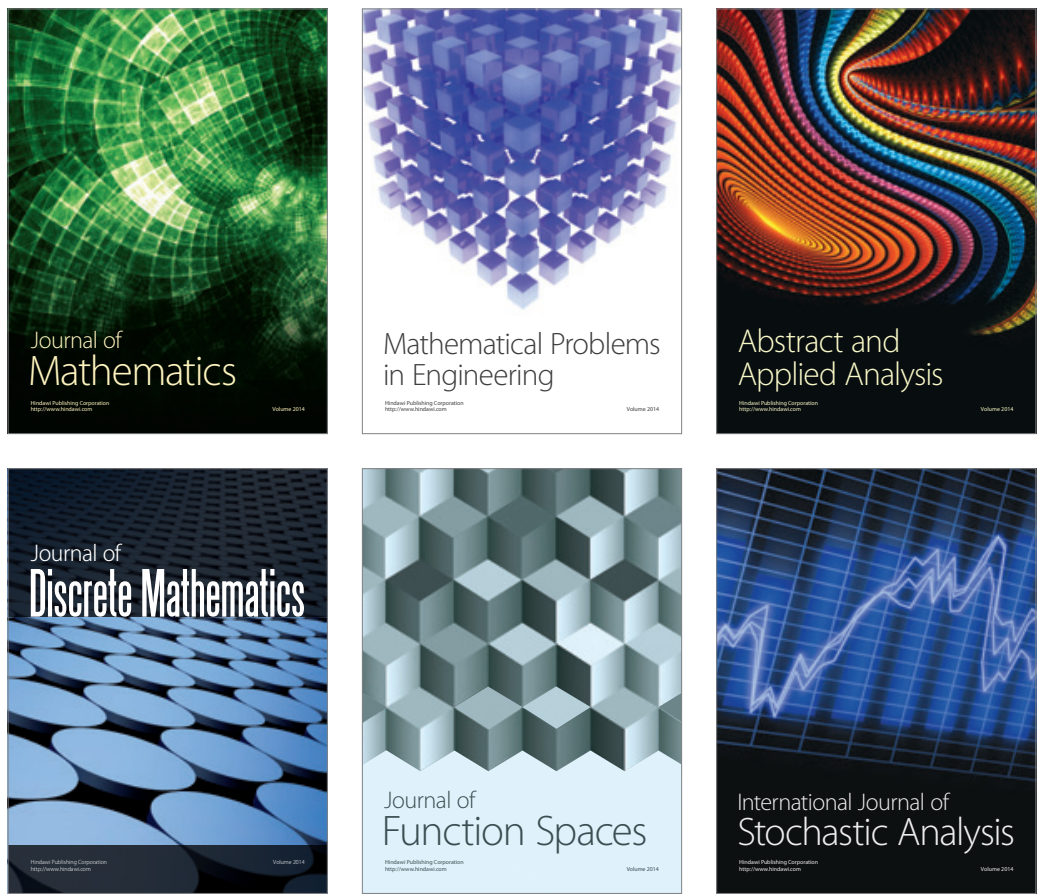

Journal of

Function Spaces

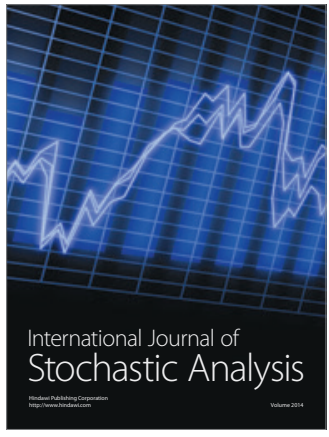

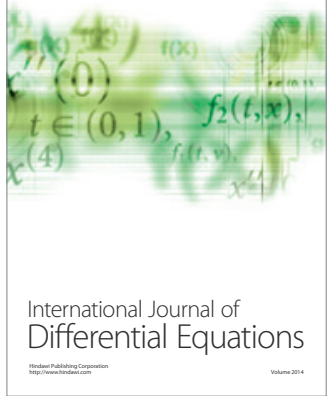
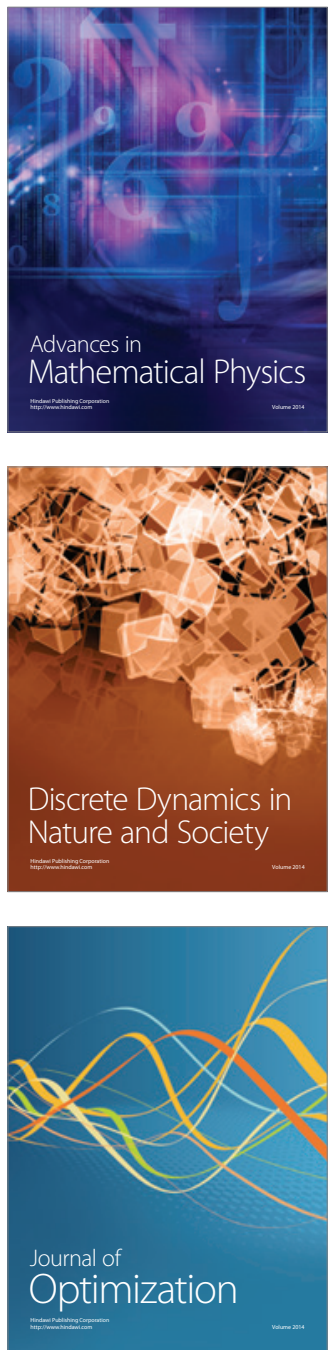